\title{
Input Zone-Selective Dysrhythmia in Motor Thalamus after Dopamine Depletion
}

\author{
Kouichi C. Nakamura, ${ }^{1}$ Andrew Sharott, ${ }^{1}{ }^{\circledR}$ Takuma Tanaka, ${ }^{2}$ and Peter J. Magill ${ }^{1,3}$ \\ ${ }^{1}$ Medical Research Council Brain Network Dynamics Unit, Nuffield Department of Clinical Neurosciences, University of Oxford, Oxford, OX1 3TH, \\ United Kingdom, ${ }^{2}$ Center for Data Science Education and Research, Shiga University, Hikone, Shiga 522-8522, Japan, and ${ }^{3}$ Oxford Parkinson's \\ Disease Centre, University of Oxford, Oxford, OX1 3QX, United Kingdom
}

The cerebral cortex, basal ganglia and motor thalamus form circuits important for purposeful movement. In Parkinsonism, basal ganglia neurons often exhibit dysrhythmic activity during, and with respect to, the slow $(\sim 1 \mathrm{~Hz})$ and beta-band $(15-30 \mathrm{~Hz})$ oscillations that emerge in cortex in a brain state-dependent manner. There remains, however, a pressing need to elucidate the extent to which motor thalamus activity becomes similarly dysrhythmic after dopamine depletion relevant to Parkinsonism. To address this, we recorded single-neuron and ensemble outputs in the basal ganglia-recipient zone (BZ) and cerebellar-recipient zone (CZ) of motor thalamus in anesthetized male dopamine-intact rats and 6-OHDA-lesioned rats during two brain states, respectively defined by cortical slow-wave activity and activation. Two forms of thalamic input zone-selective dysrhythmia manifested after dopamine depletion: (1) BZ neurons, but not CZ neurons, exhibited abnormal phase-shifted firing with respect to cortical slow oscillations prevalent during slow-wave activity; and (2) BZ neurons, but not CZ neurons, inappropriately synchronized their firing and engaged with the exaggerated cortical beta oscillations arising in activated states. These dysrhythmias were not accompanied by the thalamic hypoactivity predicted by canonical firing rate-based models of circuit organization in Parkinsonism. Complementary recordings of neurons in substantia nigra pars reticulata suggested that their altered activity dynamics could underpin the BZ dysrhythmias. Finally, pharmacological perturbations demonstrated that ongoing activity in the motor thalamus bolsters exaggerated beta oscillations in motor cortex. We conclude that BZ neurons are selectively primed to mediate the detrimental influences of abnormal slow and beta-band rhythms on circuit information processing in Parkinsonism.

Key words: basal ganglia; electrophysiology; oscillation; Parkinson's disease; thalamus

Significance Statement

Motor thalamus neurons mediate the influences of basal ganglia and cerebellum on the cerebral cortex to govern movement. Chronic depletion of dopamine from the basal ganglia causes some symptoms of Parkinson's disease. Here, we elucidate how dopamine depletion alters the ways motor thalamus neurons engage with two distinct oscillations emerging in cortico-basal ganglia circuits in vivo. We discovered that, after dopamine depletion, neurons in the thalamic zone receiving basal ganglia inputs are particularly prone to becoming dysrhythmic, changing the phases and/or synchronization (but not rate) of their action potential firing. This bolsters cortical dysrhythmia. Our results provide important new insights into how aberrant rhythmicity in select parts of motor thalamus could detrimentally affect neural circuit dynamics and behavior in Parkinsonism.

Received Aug. 27, 2021; revised Sep. 19, 2021; accepted Sep. 23, 2021.

Author contributions: K.C.N., A.S., T.T., and P.J.M. designed research; K.C.N. performed research; K.C.N., A.S., and T.T. contributed unpublished reagents/analytic tools; K.C.N. and A.S. analyzed data; K.C.N. wrote the first draft of the paper; K.C.N., A.S., T.T., and P.J.M. edited the paper; K.C.N. and P.J.M. wrote the paper.

This work was supported by Medical Research Council Awards MC_UU_12020/5, MC_UU_12024/2, and MC_UU_00003/5 to P.J.M. and Awards MC_UU_12024/1 and MC_UU_00003/6 to A.S.; Parkinson's UK Grant G-0806 to P.J.M.; and Japan Society for the Promotion of Science Grants-in-Aid for Scientific Research (KAKENHI) $15 \mathrm{H} 01663$ and 26430015. K.C.N. was supported in part by Human Frontier Science Program LT000396/2009-L. A.S. was supported in part by Marie Curie European Re-integration Grant SNAP-PD awarded by the European Union. We thank T. Kaneko for gifts of antibodies; I. Bar-Gad for MATLAB code; N. Mallet for assistance in early stages of this work; R.W. Guillery, E. Kuramoto, F. Vinciati, and H. Cagnan for insightful scientific discussions; and L. Conyers, J. Westcott, and B. Micklem for technical support.

The authors declare no competing financial interests.

Correspondence should be addressed to Kouichi C. Nakamura at kouichi.nakamura@ndcn.ox.ac.uk or Peter J. Magill at peter.magill@ndcn.ox.ac.uk.

https://doi.org/10.1523/JNEUROSCl.1753-21.2021

Copyright $\odot 2021$ Nakamura et al.

This is an open-access article distributed under the terms of the Creative Commons Attribution 4.0 International license, which permits unrestricted use, distribution and reproduction in any medium provided that the original work is properly attributed. 


\section{Introduction}

Chronic depletion of dopamine from basal ganglia (BG) circuits, as occurs in Parkinson's disease (PD), profoundly alters the electrical activities of neurons therein. Disturbed BG outputs should have detrimental consequences for their target neurons in the socalled motor thalamus (DeLong, 1990; Rubin et al., 2012; BoschBouju et al., 2013). Because motor thalamic neurons are key effectors of BG outputs, some behavioral impairments in PD likely stem from their aberrant activity dynamics.

Dysrhythmic neuronal activity (i.e., abnormal oscillatory firing) is common in BG-thalamocortical circuits in Parkinsonism (Hammond et al., 2007; Galvan et al., 2015). Neuronal dysrhythmia manifests during sleep and waking states (as well as in general anesthesia), across oscillations with markedly different frequencies. Two exemplary dysrhythmias are associated with slow oscillations $(\sim 1 \mathrm{~Hz})$ and beta-band oscillations $(\beta$ : 15 $30 \mathrm{~Hz}$ ). After dopamine depletion, many BG neurons inappropriately pattern their firing with respect to cortical slow oscillations (Magill et al., 2001; Belluscio et al., 2003; Walters et al., 2007). This might be relevant for the altered slow-wave sleep observed in people with PD (Zahed et al., 2021). However, it is unclear whether similar dysrhythmias arise in the Parkinsonian motor thalamus, hindering understanding of the wider functional impact of BG aberrations.

Studies of idiopathic PD suggest that beta-band dysrhythmia in the BG underpins bradykinesia/rigidity (Kühn et al., 2006, 2009; Ray et al., 2008; Sharott et al., 2014). Experiments in animals show that the emergence of excessive $\beta$ rhythms throughout the dopamine-depleted BG is accompanied by abnormal increases or decreases in the average firing rates of constituent neurons (Mallet et al., 2008a,b; Avila et al., 2010; Abdi et al., 2015; Sharott et al., 2017); these firing rate changes corroborate the predictions of the "direct/indirect pathways" model of BG organization in Parkinsonism (Smith et al., 1998). This influential model further posits that, because GABAergic BG output neurons are hyperactive, motor thalamus neurons are hypoactive in Parkinsonism. However, reports suggest hyperactivity (BoschBouju et al., 2014), hypoactivity (Schneider and Rothblat, 1996; Devergnas et al., 2016), or no change in firing rates in motor thalamus (Pessiglione et al., 2005; Anderson et al., 2015). One potential confounding factor is that motor thalamus is organized into discrete input zones, that is, a BG-recipient zone (BZ) and a cerebellar-recipient zone (CZ) (Nakamura et al., 2014). This important consideration aside, dopamine depletion alters other activity metrics in motor thalamus, including burst firing, pairwise correlations, and oscillatory firing (Schneider and Rothblat, 1996; Pessiglione et al., 2005; Bosch-Bouju et al., 2014; Devergnas et al., 2016). Several of these alterations show that, like the BG, the motor thalamus is dysrhythmic in Parkinsonism. A single study of motor thalamus in dopamine-depleted rats suggests that this might extend to beta-band dysrhythmia (Brazhnik et al., 2016). Critically, whether exaggerated $\beta$ oscillations are accompanied by changes in BZ neuron firing rates is unknown, as is the extent to which BZ neuronal ensembles might rhythmically synchronize their firing.

Elucidating the functional organization of the motor thalamus as a whole has benefited from direct comparisons of activity dynamics in the BZ and CZ, in both health and Parkinsonism (Anderson and Turner, 1991; Vitek et al., 1994; Guehl et al., 2003; Pessiglione et al., 2005; Ushimaru et al., 2012; Nakamura et al., 2014). The cerebellum might contribute to some compromised behaviors in PD (Wu and Hallett, 2013; Wichmann, 2019), and it is likely that CZ neuron activity is altered to some extent in Parkinsonism (Galvan et al., 2015). Because CZ is innervated by motor cortical regions exhibiting Parkinsonian $\beta$ oscillations (Mallet et al., 2008a,b), dopamine depletion might also induce beta-band dysrhythmia in CZ (Basha et al., 2014).

To resolve these issues, we quantified the brain state-dependent firing of single cells and neuronal ensembles recorded in the anatomically defined BZ and CZ of anesthetized dopamineintact and dopamine-depleted rats. Our results emphasize that motor thalamus neurons are not hypoactive in Parkinsonism, but nevertheless engage in abnormal oscillatory activities in an input zone-selective manner.

\section{Materials and Methods}

All experimental procedures were performed on adult male Sprague Dawley rats (Charles River) and were conducted in accordance with Animals (Scientific Procedures) Act, 1986 (United Kingdom). All experimental work adhered to Society for Neuroscience Policies on the Use of Animals in Neuroscience Research.

6-OHDA lesions of midbrain dopamine neurons. Unilateral 6OHDA lesions were induced in 190-250 g rats, as previously detailed (Mallet et al., 2008a,b; Abdi et al., 2015; Sharott et al., 2017). Briefly, the neurotoxin 6-OHDA (hydrochloride salt; Sigma) was dissolved in $0.9 \%$ w/v ice-cold $\mathrm{NaCl}$ solution containing $0.02 \% \mathrm{w} / \mathrm{v}$ ascorbate to a final concentration of $12 \mathrm{mg} / \mathrm{ml}$. Approximately $25 \mathrm{~min}$ before the injection of 6-OHDA, all animals received desipramine ( $25 \mathrm{mg} / \mathrm{kg}$, i.p.; Sigma) to minimize the uptake of 6-OHDA by noradrenergic neurons. Anesthesia was induced and maintained with $1.5 \%-3 \% \mathrm{v} / \mathrm{v}$ isoflurane in $\mathrm{O}_{2}$, and animals were placed in a stereotaxic frame (Kopf). Body temperature was maintained at $37 \pm 0.5^{\circ} \mathrm{C}$ by a homeothermic heating device (Harvard Apparatus). Under stereotaxic control, $1 \mu \mathrm{l}$ of 6-OHDA solution was injected near the medial forebrain bundle ( $4.1 \mathrm{~mm}$ posterior and 1.2-1.4 $\mathrm{mm}$ lateral of bregma, and $7.9 \mathrm{~mm}$ ventral to the dura) (Paxinos and Watson, 2007). Lesions were assessed 14-16 d after 6-OHDA injection by challenge with apomorphine $(0.05 \mathrm{mg} / \mathrm{kg}$, s.c.; Sigma) (Schwarting and Huston, 1996), and were considered successful when animals made $\geq 80$ net contraversive rotations in $20 \mathrm{~min}$ (Sharott et al., 2017). Electrophysiological recordings (see below) were conducted in the thalamus or substantia nigra pars reticulata $(\mathrm{SNr})$ ipsilateral to 6-OHDA lesions in anesthetized rats $21-51 \mathrm{~d}$ after surgery. The time between apomorphine administration and electrophysiological recordings was thus 7-35 d.

In vivo electrophysiological recording and juxtacellular labeling of individual thalamic neurons. Recording and labeling experiments were performed in 36 anesthetized dopamine-intact rats (300-460 g) and 10 anesthetized 6-OHDA-lesioned rats (275-416 $\mathrm{g}$ at the time of recording), as previously described (Mallet et al., 2008a,b, 2012; Nakamura et al., 2014; Abdi et al., 2015; Sharott et al., 2017). Briefly, anesthesia was induced with $4 \% \mathrm{v} / \mathrm{v}$ isoflurane in $\mathrm{O}_{2}$, and maintained with urethane (1.3 g/kg, i.p.; ethyl carbamate, Sigma), and supplemental doses of ketamine $(30 \mathrm{mg} / \mathrm{kg}$, i.p.; Willows Francis) and xylazine $(3 \mathrm{mg} / \mathrm{kg}$, i.p.; Bayer). Wound margins were infiltrated with local anesthetic $(0.5 \% \mathrm{w} / \mathrm{v}$ bupivacaine, Astra). Animals were then placed in a stereotaxic frame (Kopf). Body temperature was maintained at $37 \pm 0.5^{\circ} \mathrm{C}$ by a homeothermic heating device (Harvard Apparatus). Electrocorticograms (ECoGs) and respiration rate were monitored constantly to ensure the animals' well-being. An epidural ECoG was recorded with a 1-mm-diameter screw above the frontal (somatic sensory-motor) cortex (4.2 $\mathrm{mm}$ rostral and $2.0 \mathrm{~mm}$ lateral of bregma) (Paxinos and Watson, 2007) ipsilateral to thalamic/nigral recordings, and was referenced against a screw implanted above the ipsilateral cerebellum (Nakamura et al., 2014; Sharott et al., 2017). Raw ECoG was bandpass filtered $(0.3-1500 \mathrm{~Hz}$, $-3 \mathrm{~dB}$ limits) and amplified (2000x; DPA-2FS filter/amplifier, NPI Electronic Instruments) before acquisition. Extracellular recordings of single-unit activity, that is, the action potentials (spikes) fired by individual neurons, in the thalamus were made using standard-wall borosilicate glass electrodes (10-25 $\mathrm{m} \Omega$ in situ; tip diameter 1.0-2.0 $\mu \mathrm{m}$ ) containing 
$0.5 \mathrm{M} \mathrm{NaCl}$ solution and neurobiotin $(1.5 \% \mathrm{w} / \mathrm{v}$; Vector Laboratories, RRID:AB_2313575). Electrodes were lowered into the brain under stereotaxic guidance and using a computer-controlled stepper motor (IVM-1000; Scientifica), which allowed electrode placements to be made with submicron precision. Electrode signals were amplified $(10 \times)$ through the bridge circuitry of an Axoprobe-1A amplifier (Molecular Devices), AC-coupled, amplified another $100 \times$, and filtered at 300$5000 \mathrm{~Hz}$ (DPA-2FS filter/amplifier). The ECoG and single-unit activity were each sampled at $17.9 \mathrm{kHz}$ using a Power1401 Analog-Digital converter and a PC running Spike2 acquisition and analysis software (Cambridge Electronic Design). As described previously (Nakamura et al., 2014), single-unit activity in the thalamus was recorded during cortical slow-wave activity (SWA), which is similar to activity observed during natural sleep, and/or during episodes of spontaneous "cortical activation," which contain patterns of activity that are more analogous to those observed during the awake, behaving state (Steriade, 2000). It is important to note that the neuronal activity patterns present under this anesthetic regimen may only be qualitatively similar to those present in the unanesthetized brain. The use of supplemental doses of ketamine, an NMDAR antagonist, could have a specialized impact on brain activity dynamics. Alternative anesthetic regimens would likely result in different activity dynamics that might or might not more faithfully recapitulate those present in the unanesthetized brain. Nevertheless, the urethane-anesthetized animal (with or without supplemental ketamine) still serves as a useful model for assessing the impact of extremes of brain state on functional connectivity within and between the BG, thalamus, and cortex in dopamine-intact and Parkinsonian animals (Magill et al., 2006; Mallet et al., 2008a,b; Sharott et al., 2012, 2017; Nakamura et al., 2014). Importantly, excessive $\beta$ oscillations arise (in a brain state-dependent manner) in the BG and motor cortex of 6-OHDA-lesioned rats under this anesthetic regimen (Mallet et al., 2008a,b, 2012; Abdi et al., 2015; Sharott et al., 2017). Cortical activation was occasionally elicited by pinching a hindpaw for a few seconds. We did not analyze neuronal activity recorded concurrently with the delivery of these sensory stimuli. Because the analyzed activity was recorded at least several minutes after the cessation of the brief pinch stimulus, it was also considered to be spontaneous (Mallet et al., 2008a; Nakamura et al., 2014; Sharott et al., 2017). The animals did not exhibit a marked change in respiration rate, and did not exhibit a hindpaw withdrawal reflex, in response to the pinch. Moreover, withdrawal reflexes were not present during episodes of prolonged cortical activation, thus indicating anesthesia was adequate throughout recordings.

Following electrophysiological recordings, some single thalamic neurons were juxtacellularly labeled with neurobiotin (Lacey et al., 2007; Nakamura et al., 2014). Briefly, positive current pulses (2-10 nA, $200 \mathrm{~ms}$, $50 \%$ duty cycle) were applied until the single-unit activity became robustly entrained by the pulses. Single-unit entrainment resulted in just one neuron being labeled with neurobiotin. Two to six hours after labeling, animals were killed with an overdose of anesthetic and transcardially perfused with $100 \mathrm{ml}$ of $0.05 \mathrm{M}$ PBS, pH 7.4, followed by $300 \mathrm{ml}$ of $4 \% \mathrm{w} /$ v PFA in $0.1 \mathrm{M} \mathrm{PB}, \mathrm{pH}$ 7.4. Brains were left overnight in fixative at $4^{\circ} \mathrm{C}$, and then stored for $1-3 \mathrm{~d}$ in $\mathrm{PBS}$ at $4^{\circ} \mathrm{C}$ before sectioning (see below). Seventy-nine of the thalamic neurons detailed herein were juxtacellularly labeled. These neurobiotin-labeled neurons were designated "identified" and precisely localized to different input zones of the motor thalamus (see below). The remaining (unlabeled) thalamic neurons $(n=100)$ were also included because, using stereotaxy and readouts from the stepper motor, we could accurately extrapolate their locations from those of identified neurons (recorded with the same glass electrodes in the same animals). Henceforth, we designate these unlabeled neurons as "extrapolated." The identified and extrapolated thalamic neurons recorded in dopamine-intact rats with glass electrodes are those reported by Nakamura et al. (2014), but their firing properties have now been reanalyzed to address the issues underpinning the current study; specifically, we have performed new analysis of their firing with respect to ongoing cortical $\beta$ oscillations, and we have statistically compared thalamic neuron firing rates/patterns in dopamine-intact rats versus 6-OHDA-lesioned rats.

In vivo electrophysiological recording of thalamic and nigral activity with multielectrode arrays. Extracellular "wideband" $(0.1-6000 \mathrm{~Hz})$ recordings of neuronal activity were simultaneously made from numerous sites in the motor thalamus or $\mathrm{SNr}$ of urethane-anesthetized dopamine-intact rats $(n=7 ; 295-385 \mathrm{~g})$ and 6-OHDA-lesioned rats $(n=11$; $300-500 \mathrm{~g}$ at the time of recording) using linear electrode arrays with multiple, spatially-defined recording contacts ("silicon probes"; A1x16$10 \mathrm{~mm}-100-400$ or A1x16-10 mm-100-177, NeuroNexus), as previously described (Magill et al., 2006; Mallet et al., 2008b; Sharott et al., 2017). Each probe had 16 recording contacts arranged in a single vertical plane, with a contact separation of $100 \mu \mathrm{m}$. Depending on the probe used, each contact had an area of $\sim 400 \mu \mathrm{m}^{2}$ (impedance of 0.9-1.2 $\mathrm{M} \Omega$, measured at $1000 \mathrm{~Hz}$ ) or $177 \mu \mathrm{m}^{2}$ (impedance of 1.7-2.0 $\mathrm{M} \Omega$ ). To enable post hoc histological verification of recording sites (see below), the backs of the silicon probes were evenly coated before each experiment with the red fluorescent dye DiI (D3911, Invitrogen) by application of a $100 \mathrm{mg} / \mathrm{ml}$ DiI solution in acetone (Magill et al., 2006). A single probe was manually advanced into the brain using a zero-drift micromanipulator (1760-61, Kopf) under stereotaxic control. The probes were cleaned after each experiment in a proteolytic enzyme solution (Magill et al., 2006). This was sufficient to ensure that contact impedances and recording performance were not altered by probe use and reuse. Monopolar probe signals were recorded using high-impedance unity-gain operational amplifiers (Advanced LinCMOS, Texas Instruments) and were referenced against a screw implanted above the contralateral cerebellum. After initial amplification, extracellular signals were further amplified $(1000 \times)$ and low-pass filtered at $6000 \mathrm{~Hz}$ using programmable differential amplifiers (Lynx-8, Neuralynx). ECoGs were also recorded as described above. The probe signals and ECoGs were each sampled at $17.9 \mathrm{kHz}$ using a Power 1401 converter and a PC running Spike2 software. After the recording sessions, animals were killed and transcardially perfused with fixative (as described above) for post hoc histological analyses.

Microinfusions of GABA into thalamus. For these experiments, urethane-anesthetized 6-OHDA-lesioned rats $(n=10 ; 300-500 \mathrm{~g}$ at the time of recording) were prepared for ECoG recordings as described above. A glass micropipette (tip diameter $\sim 30 \mu \mathrm{m}$ ) containing a $0.5 \mathrm{M}$ GABA solution (0344, Tocris Bioscience; dissolved in $0.9 \% \mathrm{w} / \mathrm{v} \mathrm{NaCl}$ solution) was then advanced into the brain using a zero-drift micromanipulator under stereotaxic control. During sustained periods of cortical activation ( $>100 \mathrm{~s}$ of ensuing $\beta$ oscillations in ECoGs), $60 \mathrm{nl}$ of the GABA solution was slowly infused (mean duration [ \pm SEM]: $24.4 \pm 1.0 \mathrm{~s}$ ) into the motor thalamus using air pressure under manual control. Because the inactivation effects of such GABA microinfusions typically wear off within a few minutes (Kojima and Doupe, 2009), we were able to perform repeated infusions (using a minimal interval of $10 \mathrm{~min}$ ) at one or more thalamic sites in a single animal; this also allowed us to negate the possibility of a spontaneous disappearance of cortical $\beta$ oscillations. To mark the microinfusion sites at the end of each experiment, 100-200 nl of a $0.9 \% \mathrm{w} / \mathrm{v} \mathrm{NaCl}$ solution containing $0.04 \% \mathrm{w} / \mathrm{v}$ blue fluorescent microspheres (F8797, Invitrogen) was infused at the same stereotaxic coordinates through the same micropipette, as described previously (Nakamura and Morrison, 2007). Animals were then killed and transcardially perfused with fixative for post hoc histological analyses.

Histology, immunofluorescence, and microscopy. The fixed brains were cut into $50-\mu \mathrm{m}$-thick sections in the parasagittal plane on a vibrating microtome (VT1000S, Leica Microsystems), collected in series, and washed in PBS. For anatomical analyses of the brains of 6-OHDAlesioned rats in which thalamic recordings had been performed, parasagittal sections ipsilateral and contralateral to the site of 6-OHDA injection were bisected just rostral to the SNr. The rostral half-sections were processed to reveal markers of relevance to the motor thalamus, whereas the caudal half-sections were processed for markers of relevance to the midbrain (i.e., substantia nigra pars compacta $(\mathrm{SNc})$ and $\mathrm{SNr}$ ). For anatomical analyses of 6-OHDA-lesioned rats in which only nigral recordings were performed, parasagittal sections were kept intact before revealing markers of relevance to the $\mathrm{SNc}$ and $\mathrm{SNr}$. All the following reagent incubations were performed at room temperature.

To qualitatively assess the extent of 6-OHDA lesions in the midbrain, tissue sections ipsilateral and contralateral to the site of 6-OHDA injection were washed in PBS and then processed to reveal immunoreactivity for tyrosine hydroxylase (TH), a marker of dopaminergic neurons. 
Sections were incubated overnight with a primary antibody mixture of rabbit anti-TH (1:1000 dilution; AB152, Millipore, RRID:AB_390204) and mouse anti-glutamic acid decarboxylase of $67 \mathrm{kDa}$ (GAD67; $2 \mu \mathrm{g} /$ ml; MAB5406, Millipore, RRID:AB_2278725) in Triton-PBS (PBS with $0.3 \% \mathrm{v} / \mathrm{v}$ Triton X-100 [Sigma]) containing $1 \% \mathrm{v} / \mathrm{v}$ donkey serum (Jackson ImmunoResearch Laboratories; all the following antibody incubations were conducted with the same buffer). After washing with Triton-PBS, the sections were incubated for 2-4 $\mathrm{h}$ with a mixture of fluorophore-conjugated secondary antibodies (all raised in donkey): antirabbit IgG (Alexa Fluor 488; 1:500; Invitrogen) and anti-mouse IgG (Alexa Fluor 647; 1:200; ImmunoResearch Laboratories). After washing, the fluorescently labeled sections were mounted on glass slides, coverslipped, and examined with a laser-scanning confocal microscope (LSM710, Carl Zeiss).

To visualize neurobiotin-filled neurons, free-floating sections were washed in PBS and incubated overnight in Cy3-conjugated streptavidin (1:1000; PA43001, GE Healthcare) in Triton-PBS. After washing, the sections were mounted on glass slides, coverslipped, and examined with an epifluorescence microscope (AxioPhot, Carl Zeiss) to identify the neurobiotin-filled neurons. To delineate thalamic nuclei and map the location of each identified neuron, sections containing the fluorescently-labeled somata were subsequently incubated overnight with a primary antibody mixture of rabbit anti-vesicular glutamate transporter 2 (VGluT2; $0.4 \mu \mathrm{g} / \mathrm{ml}$ of affinity-purified IgG, a gift from Prof. Takeshi Kaneko, Kyoto University) (Hioki et al., 2003) and mouse anti-GAD67 $(2 \mu \mathrm{g} / \mathrm{ml}$; MAB5406, Millipore, RRID:AB_2278725) in Triton-PBS containing 1\% $\mathrm{v} / \mathrm{v}$ donkey serum (all the following antibody incubations were conducted with the same buffer). After washing with Triton-PBS, the sections were incubated for 2-4 h with a mixture of fluorophore-conjugated secondary antibodies (all raised in donkey): anti-rabbit IgG (DyLight 649; 1:200; ImmunoResearch Laboratories) and anti-mouse IgG (Alexa Fluor 488; 1:200; Invitrogen). When necessary, the adjacent sections were incubated with NeuroTrace 500/525 (1:150; N-21 480, Invitrogen), a green fluorescent Nissl stain, in Triton-PBS for $30 \mathrm{~min}$ to visualize cytoarchitecture. After washing, the fluorescently labeled sections were mounted on glass slides, coverslipped, and examined with a laser-scanning confocal microscope (LSM710, Carl Zeiss). To precisely localize the recorded neurons to distinct thalamic nuclei, fluorescent images of the thalamus around the neurobiotin-filled neurons were taken at a low magnification with a $5 \times$ objective lens (EC Plan-Neofluar, numerical aperture 0.16; Carl Zeiss), a pinhole thoroughly opened (i.e., in "nonconfocal" mode), and a zoom factor of 0.6. Appropriate sets of laser beams and emission windows were used for Alexa Fluor 488 (excitation $488 \mathrm{~nm}$, emission 492-544 nm), Cy3 (excitation $543 \mathrm{~nm}$, emission 552$639 \mathrm{~nm}$ ), and DyLight 649 (excitation $633 \mathrm{~nm}$, emission 639-757 nm). Images of each of the channels were taken separately and sequentially to negate possible "bleed through" of signal across channels. Images were combined into montages and, when necessary, images from the adjacent sections were overlaid and aligned using graphic software (Canvas 12, ACD Systems, RRID:SCR_014288). The two input zones of the motor thalamus (BZ and CZ) were delineated on the basis of their distinctive distributions of VGluT2 and GAD67 immunoreactivities (Kuramoto et al., 2009, 2011, 2015; Nakamura et al., 2014). Only identified neurons located $>50 \mu \mathrm{m}$ away from the borders of BZ or CZ were analyzed. Extrapolated neurons had to be located $>100 \mu \mathrm{m}$ away from these borders to be included in the analyses. The dendrites of thalamocortical neurons in the rat motor thalamus only rarely radiate $>200 \mu \mathrm{m}$ from the parent somata (Kuramoto et al., 2009, 2015). Thus, most of the proximal dendrites of our neurons were likely to be confined to just one zone.

To determine the locations of silicon probe recording sites (see Figs. 6 and 8), tissue sections were mounted on glass slides in PBS, coverslipped, and examined with a microscope capable of fluorescent and brightfield imaging (Axio Imager.M2, Carl Zeiss). In those sections containing DiI signal, images of fluorescence (43 Cy3 filter) and transmitted light were then taken with a $1.25 \times$ objective (EC Plan-Neofluar, numerical aperture 0.03; Carl Zeiss) to record the probe penetration tracks. In many cases, the best quality DiI images were obtained at this stage because the subsequent immunofluorescence protocol tended to "wash out" the DiI. At the start of the immunofluorescence protocol, the DiIcontaining sections were unmounted and then heat treated as a means of antigen retrieval $\left(80^{\circ} \mathrm{C}\right.$ for $30 \mathrm{~min}$ in $10 \mathrm{~mm}$ citrate- $\mathrm{NaOH}$ buffer, $\mathrm{pH}$ 6.0). After washing with PBS, the sections were incubated overnight with a primary antibody mixture of guinea pig anti-glycine transporter 2 (GlyT2; 1:10,000; AB1773, Merck, RRID:AB_90953) and mouse anti-GAD67 $(1 \mu \mathrm{g} / \mathrm{ml}$; MAB5406, Millipore, RRID:AB_ 2278725) for the thalamus, or mouse anti-GAD67 antibody alone for the SNr, in Triton-PBS containing 10\% v/v donkey serum (Jackson ImmunoResearch Laboratories; all the following antibody incubations were conducted with the same buffer). After washing with Triton-PBS, the sections were incubated for $2-4 \mathrm{~h}$ with a mixture of fluorophore-conjugated secondary antibodies (all raised in donkey, 1:500, Jackson ImmunoResearch Laboratories): anti-guinea pig IgG (DyLight 488) and anti-mouse IgG (Alexa Fluor 647) for the thalamus, or anti-mouse IgG (Alexa Fluor 647) alone for the SNr. A laserscanning confocal microscope was used to take low-magnification fluorescent images of the thalamus around the DiI signal, as described above. To correct for tissue shrinkage $(<10 \%)$ that occurred during the immunofluorescence protocol, images taken after immunofluorescence were scaled and aligned with images taken before immunofluorescence, using Canvas 12 software. Judging from the known stereotaxic distances between probe penetrations in a single plane (see Figs. $6 A, A^{\prime}$ and $8 A, A^{\prime}$ ), we estimated the difference between the scaling in the tissue in vivo during recordings and the scaling in the images before immunofluorescence to be $<10 \%$. The most ventral DiI deposit in each penetration track was considered to be the location of the probe tip; extrapolating from this, the estimated positions of the probe recording contacts were plotted on the images of DiI signal and immunofluorescence (see Figs. $6 A, A^{\prime}$ and $8 A, A^{\prime}$ ). Thalamic nuclei/zones and the $\mathrm{SNr}$ were identified according to immunofluorescence images, and each recording contact was assigned a location tag for group analyses. When the estimated locations of probe contacts were on or close to $(\leq 50 \mu \mathrm{m})$ the borders between structures, those recording sites were tagged as "border" and were excluded from group analyses (Figs. $6 B-D$ and $8 B-D$ ).

To determine the sites of GABA microinfusion, we used an anatomical analysis pipeline similar to that for localizing silicon probes, with the key difference being that glass pipette tracks were visualized with blue fluorescent microspheres (see Fig. 10). Thalamic nuclei/zones were identified with immunofluorescence for GlyT2, VGluT2, and GAD67, using the primary antibodies described above and revealed with secondary antibodies that were conjugated to DyLight 488, Cy3, and Alexa Fluor 647 , respectively. A laser-scanning confocal microscope was first used to take low-magnification fluorescent images of the blue microspheres (excitation $405 \mathrm{~nm}$, emission $409-559 \mathrm{~nm}$ ) in the tissue. Using the same objective and zoom, images of DyLight 488, Cy3, and Alexa Fluor 647 signals were then taken sequentially and separately (as above) to map thalamic structures. After registration of images, the ends of the pipette tracks (considered to be the locations of the pipette tips) were assigned a location tag.

Analysis of ECoGs and basic firing parameters of single units. ECoG data from each recording session were visually inspected and epochs of robust cortical SWA or cortical activation were selected according to the previously described characteristics of these brain states (Mallet et al., 2008b; Nakamura et al., 2014; Sharott et al., 2017). A 100 s portion of the glass electrode or silicon probe data concomitantly recorded during each defined brain state was isolated and used for statistical analyses. Silicon probe data were high-pass filtered off-line at $300 \mathrm{~Hz}$ (Spike2, finite impulse response filter) to isolate unit activity. Putative single-unit activity was isolated with standard "spike sorting" procedures (Mallet et al., 2008a), including template matching, principal component analysis, and supervised clustering (Spike2). Isolation of a single unit was verified by the presence of a distinct refractory period in the interspike interval (ISI) histogram. Only neurons in which $<1 \%$ of all ISIs were $<2 \mathrm{~ms}$ were analyzed in this study. Single-unit activity was converted so that each spike was represented by a single digital event (Spike2). The recorded signals and sorted spike trains were then resampled at $17 \mathrm{kHz}$ with Spike2 and exported to MATLAB (The MathWorks, RRID:SCR_001622) for further 
analysis. Spike trains were assumed to be realizations of stationary stochastic point processes. The mean firing rate (spikes/s) of individual neurons was calculated from the total number of spikes per $100 \mathrm{~s}$ data epoch. Variability of firing was assessed using a metric related to the coefficient of variation (CV) of the ISI, the mean CV2 (Holt et al., 1996); the lower the CV2 value, the more regular the unit activity.

Detection of low-threshold $\mathrm{Ca}^{2+}$ spike (LTS) bursts fired by thalamic neurons. LTS bursts were classified as such using custom Spike2 scripts, according to previously defined criteria for identifying LTS bursts in extracellular unit recordings (Lacey et al., 2007; Nakamura et al., 2014): (1) at least 2 action potentials with an ISI of $\leq 5 \mathrm{~ms}$ but with a preceding silent period of $>100 \mathrm{~ms}$ (Lu et al., 1992); and (2) a maximum ISI of $10 \mathrm{~ms}$ was used to define the end of a LTS burst (Fanselow et al., 2001).

Detection of the burst firing of SNr neurons. Analyses of $\mathrm{SNr}$ neuron burst firing in lesioned rats was conducted on single units that displayed high variability in their ISIs $(\mathrm{CV}>1.0)$ during SWA. To detect the onsets and offsets of burst firing, $\mathrm{SNr}$ spike trains ( $n=14$, from 5 rats) were first converted into a spike density function (SDF; see Fig. 4G) (Szucs, 1998). The point process data were thus converted to continuous time series (changes in firing probability over time) by convolution (with MATLAB function conv) of a Gaussian kernel with a $\sigma$ of $0.051 \mathrm{~s}$ (selected so that $95 \%$ of spikes are within the range of $[-3 \sigma, 3 \sigma]$ ), as prepared by MATLAB function normpdf. Peaks and troughs in the SDF waveform were then detected using MATLAB function findpeaks, using a minimal peak/trough duration of $2 \sigma$ and, for peak detection only, setting the minimal peak height to be the maxima of the Gaussian kernel. A threshold of the SDF waveform was then set as the mean of the median peaks and median troughs (see Fig. 4G). Within the epochs that the SDF waveform was equal to or higher than this threshold, the spike events that were closest to the times that the SDF rose above or fell below the threshold were considered to be the onsets and offsets of bursts, respectively.

Analysis of phase-locked firing of single units, including circular statistics. To investigate how the activity of individual thalamic and nigral neurons varied in time with respect to ongoing cortical network activity, we analyzed the instantaneous phase relationships between thalamic/ nigral spike times and cortical oscillations in specific frequency bands (Sharott et al., 2012, 2017; Nakamura et al., 2014; Garas et al., 2016). Signal analyses were performed using MATLAB. ECoG signals containing robust SWA or cortical activation were initially downsampled to $1024 \mathrm{~Hz}$ (MATLAB function resample) and then digitally bandpass filtered to isolate slow $(0.4-1.6 \mathrm{~Hz})$ or $\beta(15-30 \mathrm{~Hz})$ oscillations, respectively (second- and fifth-order zero-phase Butterworth filters for slow and $\beta$ oscillations, respectively, using MATLAB function filtfilt). Subsequently, the instantaneous phase and power of the ECoG in these frequency bands were separately calculated from the analytic signal obtained via the Hilbert transform (Lachaux et al., 1999). In this formalism, peaks in the ECoG oscillations correspond to a phase of $0^{\circ}$ and troughs to a phase of $180^{\circ}$. Linear phase histograms, circular phase plots, and circular statistical measures were calculated using the instantaneous phase values for each spike. Descriptive and inferential circular statistics were then calculated using the CircStat toolbox (Berens, 2009) for MATLAB. The phase-locked firing of each neuron with respect to cortical oscillations was represented by a vector whose angle and length (bound between 0 and 1 ; the closer to 1 , the more concentrated the angles) were, respectively, defined as the circular mean (circ_mean of CircStat) and the mean resultant vector length (simply referred to as 'vector length'; circ_r of CirtStat) of the instantaneous phase values of spikes. For the calculation of vector lengths and statistical comparisons, we included only those neurons that fired $\geq 40$ spikes during the entire analyzed epoch (100 s). These "qualifying" neurons were then tested for significantly phase-locked firing (defined as having $p<0.05$ in Rayleigh's Uniformity Test; circ_rtest of CircStat). The null hypothesis for Rayleigh's test was that the spike data were distributed in a uniform manner across/throughout phase. We and others have previously remarked that the nonsinusoidal nature of some field potential oscillations, such as the cortical slow oscillation, can confound standard circular statistics, especially Rayleigh's test (Siapas et al., 2005; Mallet et al., 2008a, 2008b; Sharott et al., 2012; Nakamura et al., 2014). Thus, for analysis of neuron firing relationships with cortical slow oscillations, Rayleigh's tests were only conducted after any phase nonuniformities of the slow oscillations were corrected with the empirical cumulative distribution function (MATLAB ecdf) (Siapas et al., 2005; Nakamura et al., 2014; Abdi et al., 2015; Garas et al., 2016). For each of the neurons that were significantly phase-locked using these criteria, the mean phase angle was calculated. Because some datasets did not meet the requirements for parametric testing, differences in the median phase angles of groups of neurons were tested using the nonparametric common median test (circ_cmtest of CircStat) (Fisher, 1995). The null hypothesis for the common median test is that all tested groups have the same circular median. The vector length was used to quantify the level of phase locking around the mean phase for individual neurons (computed using the angles of each spike) and for populations of neurons (computed using the mean phase for each neuron). Where single-unit data are displayed in circular plots, lines radiating from the center are the vectors of the preferred phases of firing (with the center and perimeter of the outer grid circle representing vector lengths of 0 and 1, respectively); thin lines indicate preferred firing of individual neurons, whereas thick lines indicate population vectors. The small open circles on the perimeter represent the preferred phases of each neuron.

Extraction and conditioning of background-unit activity (BUA) signals for time-series analyses. We analyzed BUA signals recorded with silicon probes, a representation of the summed firing of small, local neuronal populations that is conceptually distinct from multiunit activity and LFPs (Moran and Bar-Gad, 2010). These BUA signals were isolated from the wideband recordings made with the probes by initial high-pass filtering off-line at $300 \mathrm{~Hz}$ (Spike2, finite impulse response filter) and the removal of any large-amplitude action potentials that could potentially distort the signals and bias analyses, as per Moran et al. (2008) (see Extended Data Fig. 6-1A-F). Large-amplitude action potentials were defined as those exceeding 3 SDs of the entire high-pass filtered signal, and data points around these large action potentials were removed and replaced with another randomly selected part of the recording that did not contain similarly large action potentials (MATLAB createBUA function, a gift from Dr Izhar Bar-Gad). The windows for spike removal were generally set to -1.5 and $2.0 \mathrm{~ms}$ before and after the peak of the large action potentials, with a few exceptions ( 2 and 14 of 673 channels for before and after a spike, respectively) for which wider (up to -3.0 and $3.0 \mathrm{~ms}$ ) windows were used to avoid artifacts as assessed by visual inspection. The BUA signals were then full-width rectified (Journée, 1983; Myers et al., 2003; Moran et al., 2008) and mean subtracted to remove the DC component that was created by rectification (see Extended Data Fig. 6-1G-I). Finally, the signals were low-pass filtered at $300 \mathrm{~Hz}$ (zero-phase shift Butterworth filter with the order of 3) and downsampled to $1024 \mathrm{~Hz}$ (see Extended Data Fig. 6-1J-L) before further time-series analyses.

Analysis of phase-locked BUA signals, including circular statistics. To investigate how thalamic and nigral BUA varied in time with respect to ongoing cortical $\beta$ oscillations, we analyzed "phase-averaged waveforms" of BUA signals, that is, the average of BUA voltage $(\mu \mathrm{V})$ in each bin $\left(\right.$ size $\left.=5^{\circ}\right)$ of the instantaneous phase values of the ECoG bandpass filtered at $15-30 \mathrm{~Hz}$ (zero-phase shift Butterworth filter with the order of 3). A sample vector $V$ for an individual BUA signal is defined in the complex plane as the double of an average of complex number-based vector representations of the instantaneous phase values and the values of BUA signals of each data point, i.e., $V=\frac{2}{N} \sum_{k=1}^{N} r_{k} e^{i \varphi_{k}}$, where $\varphi_{k}$ and $r_{k}$ represent the instantaneous phase in radians and the value of BUA signal (signed) in $\mu \mathrm{V}$ for the $k$ th data point, respectively, $N$ is the number of data points, and $i$ is the imaginary unit. The average was doubled to reflect the amplitude difference between the positive and negative deflections. If the phase-averaged waveform of a signal is an ideal sinusoidal curve, the sample vector length $|V|$ is identical to the peak-topeak amplitude in $\mu \mathrm{V}$.

In order to assess the nonuniformity (i.e., significant phase modulation) of BUA signals in relation to cortical $\beta$ oscillations (see Figs. $7 G, H$ and $9 G, H$ ), data points were circularly shifted in a random manner within each cycle of the $\beta$ oscillations (MATLAB circshift). This 
maintained the waveform of BUA signals within a cycle while randomizing their phase relationships to ECoG. A similar approach has been used previously (von Nicolai et al., 2014). We randomly chose 10 BUA signals in $\mathrm{BZ}$ or $\mathrm{SNr}$ and performed 1000 iterations of random shifting of each signal to generate a histogram based on an empirical cumulative distribution function of vector lengths of the shifted data (MATLAB ecdfhist). A BUA signal was considered to be "significantly modulated" in relation to ECoG $\beta$ oscillations when the sample vector length was longer than $99.9 \%$ of the sample vector lengths of the shifted data. Where significantly modulated BUA signals are displayed in circular plots (see Figs. 7I and $9 I$ ), lines radiating from the center are the vectors of their preferred phases (with the center and perimeter of the outer grid circle representing vector lengths of 0 and $1.2 / 0.8 \mu \mathrm{V}$, respectively); thin lines indicate the preferred phase of individual BUA signals, whereas thick lines indicate population vectors. The small open circles on the perimeter represent the preferred phases of each BUA signal. Group analysis of the sample vectors for BUA signals was conducted as for group analysis of single-unit data (see above).

Spectral analyses. ECoGs were downsampled to $1024 \mathrm{~Hz}$ before spectral analyses. Spectral parameters for ECoG and BUA time series were evaluated using FFT. Power and coherence spectra were calculated with MATLAB pwelch and mscohere functions, with an FFT size of 5120 for signals recorded during SWA (giving a frequency resolution of $0.2 \mathrm{~Hz}$ ) and an FFT size of 1024 for signals recorded during cortical activation ( $1.0 \mathrm{~Hz}$ resolution). The overlap of FFT windows was $50 \%$. For power spectra, each individual power spectrum was normalized to give "\% relative power" unless otherwise stated. This was achieved by calculating the spectral power in each frequency bin as a percentage of the total power between 0.4 and $100 \mathrm{~Hz}$ (for analysis of SWA) or between 1 and $100 \mathrm{~Hz}$ (for analysis of cortical activation). For statistical comparisons, the sum of power or the average of coherence across all frequency bins in the band of interest (i.e., $0.4-1.6 \mathrm{~Hz}$ or $15-30 \mathrm{~Hz}$ ) was calculated, giving a single value for each recording.

Analysis of effects of GABA microinfusion. We characterized the extent to which microinfusions of GABA into thalamus influenced ongoing cortical $\beta$ oscillations. To visualize changes in ECoG power at $\beta$ frequencies $(15-30 \mathrm{~Hz})$ over time and across experiments, we plotted spectrograms in which beta-band power was normalized to that in a preGABA period set as the $100 \mathrm{~s}$ immediately before GABA infusion onset (MATLAB spectrogram function; FFT size of 16,384, FFT window overlap of $50 \%$, frequency resolution of $0.0625 \mathrm{~Hz}$, temporal resolution of 8 s). The effect size (\%) of GABA infusion was defined as $(1-x) \times 100$ (\%), where $x$ is the ratio of the total ECoG beta-band power during the 50-150 $\mathrm{s}$ after GABA infusion onset to that during the pre-GABA period.

Experimental design and statistical analyses. For each experiment, descriptions of critical variables (e.g., number of animals, neurons, and other samples evaluated) as well as statistical design can be found in Results. The Shapiro-Wilk test (swtest by Ahmed BenSaïda) was used to judge whether noncircular datasets were normally distributed $(p<0.05$ to reject). Because some datasets were not normally distributed, we used nonparametric statistical testing for these data throughout. The MannWhitney $U$ test (MWUT; MATLAB ranksum) was used for comparisons of unpaired data. For multiple group comparisons, we performed a Kruskal-Wallis one-way ANOVA on ranks (MATLAB kruskalwallis), with Dunn's test (MATLAB multcompare) for further post hoc definition of comparisons. $H$ statistics, approximated by $\chi^{2}$, for Kruskal-Wallis ANOVAs are reported with degrees of freedom in parenthesized subscript. Significance for all statistical tests was set at $p<0.05$ (exact $p$ values are given in the text). Data are represented as group mean \pm SEM unless stated otherwise. All box plots in figures show the individual samples (circles), medians, the interquartile ranges (box), and the nonoutlier values closest to the first and third quartiles (whiskers). We used male rats, and care should be taken in extrapolating our results to females.

\section{Results}

The overall aim of this study was to define how the chronic depletion of dopamine, as occurs in PD, alters the spatial and temporal organization of electrical activity within the two major input zones of the motor thalamus in vivo. Emphasis was placed on defining the extent to which the action potential firing of $\mathrm{BZ}$ and CZ neurons becomes dysrhythmic during, and with respect to, the slow oscillations $(0.4-1.6 \mathrm{~Hz})$ and $\beta$ oscillations $(15-30 \mathrm{~Hz})$ emerging in cortico-BG circuits in a brain state- and dopamine-dependent manner. To address this, we first recorded individual, identified neurons in the $\mathrm{BZ}$ and $\mathrm{CZ}$ of anesthetized dopamine-intact rats and dopaminedepleted (6-OHDA-lesioned) rats during two well-defined and controlled brain states, SWA and cortical activation. To gain further insights into the activity dynamics of larger neuronal populations during cortical activation, we sampled background-unit activities from numerous sites in and around the BZ and CZ using linear multielectrode arrays. Further functional context was provided by recording the activities of neurons in the $\mathrm{SNr}$, one of the BG output nuclei that targets BZ, as well as by examining the effects of pharmacological perturbations of the $\mathrm{BZ}$ and $\mathrm{CZ}$.

\section{Dopamine depletion does not decrease the firing rates of neurons in the motor thalamus during cortical SWA or cortical activation}

The influential direct/indirect pathways model of BG organization in Parkinsonism predicts that motor thalamus neurons are hypoactive in Parkinsonism (DeLong, 1990; Smith et al., 1998). Ongoing brain state provides critical context when testing the validity of the model's predictions; not only are the firing rates of motor thalamus neurons in dopamine-intact rodents exquisitely dependent on brain state (Ushimaru et al., 2012; Nakamura et al., 2014), but it has also been established that dopamine depletion only alters the firing rates of BG neurons in the expected manner during certain brain states (Abdi et al., 2015; Sharott et al., 2017; Kovaleski et al., 2020). With this in mind, and throughout this study, we interrogated neuronal activity dynamics in the motor thalamus in the context of cortical SWA and cortical activation, as verified in simultaneous recordings of ipsilateral frontal ECoGs (Magill et al., 2006; Mallet et al., 2008a,b; Nakamura et al., 2014).

Using glass electrodes, we recorded the spontaneous action potential discharges (spikes) of 137 single units (neurons) in the motor thalamus of dopamine-intact control rats $(n=36)$, and 42 neurons in the motor thalamus of 6-OHDA-lesioned rats $(n=10)$, during cortical SWA and/or cortical activation. Of these neurons, $46 \%$ (63 of 137) and 38\% (16 of 42) were unequivocally identified; that is, after electrophysiological characterization, they were juxtacellularly labeled with neurobiotin, and their somata were precisely localized to the BZ or CZ (Fig. 1; Extended Data Fig. 1-1A). As described previously (Kuramoto et al., 2009, 2011; Bosch-Bouju et al., 2014; Nakamura et al., 2014), we used markers of specific groups of GABAergic axon terminals or glutamatergic axon terminals (i.e., GAD67 and VGluT2, respectively) to define the boundaries of the $\mathrm{BZ}$ and $\mathrm{CZ}$ (Fig. 1). We also anatomically verified that the 6-OHDA injections profoundly reduced the prevalence of dopaminergic neurons in the midbrain ipsilateral to injection sites (Extended Data Fig. 1-1B, $C)$. During periods of robust SWA in ipsilateral frontal cortex, the activity of identified BZ neurons in both dopamine-intact rats and lesioned rats was typified by a relatively low mean firing rate $(<4$ spikes/s) and a propensity to fire discrete bursts of spikes (Fig. $1 A, B$ ). These bursts were exemplified by 2-6 spikes fired in rapid succession (instantaneous intraburst rates of $>150$ spikes/s), with a progressive decrease in spike amplitude (Fig. 


\section{Dopamine-Intact}
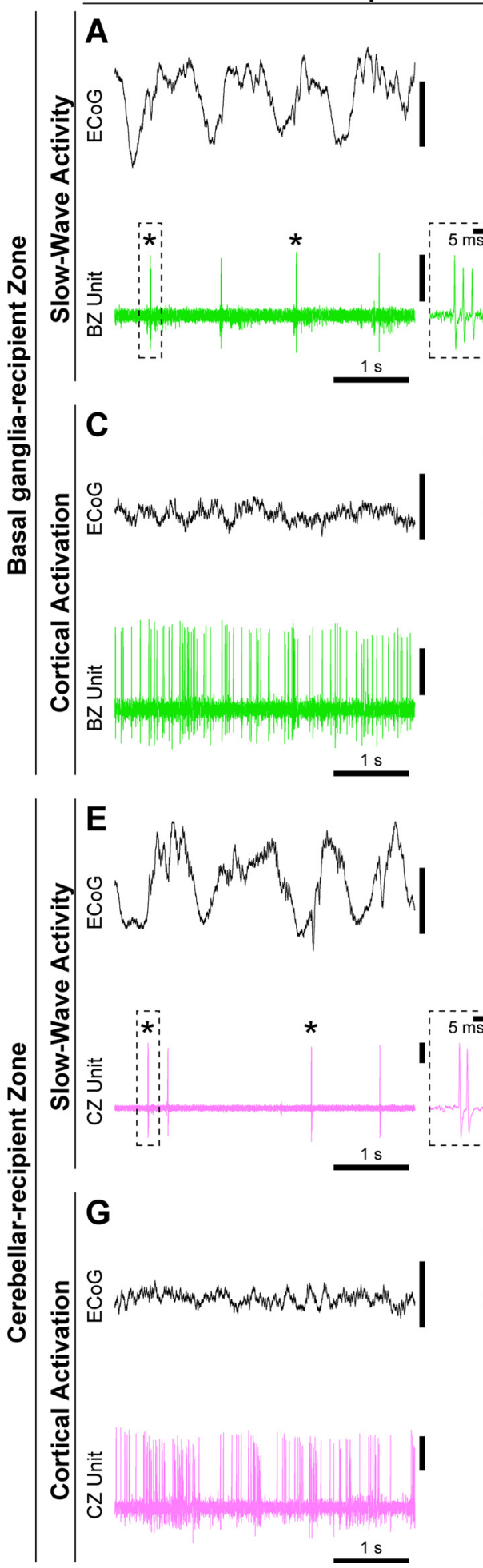
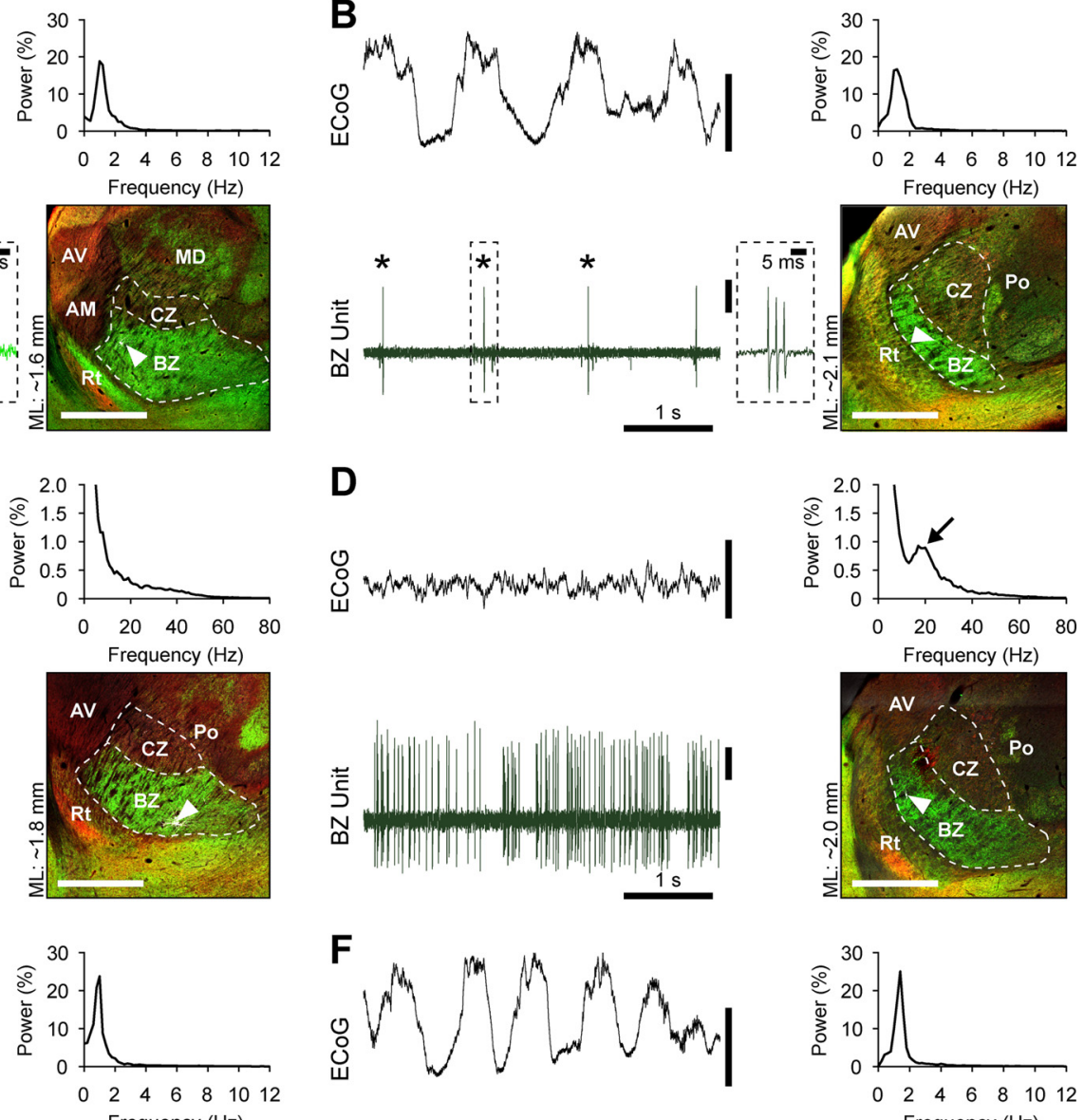

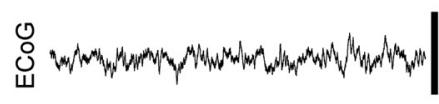
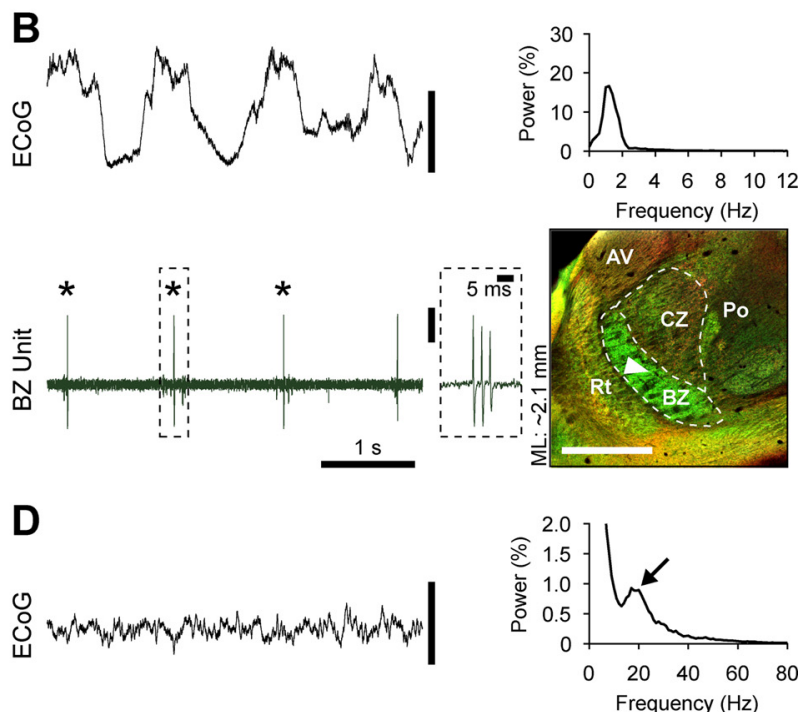

D

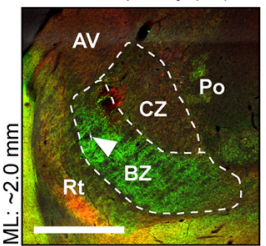
Frequency $(\mathrm{Hz})$

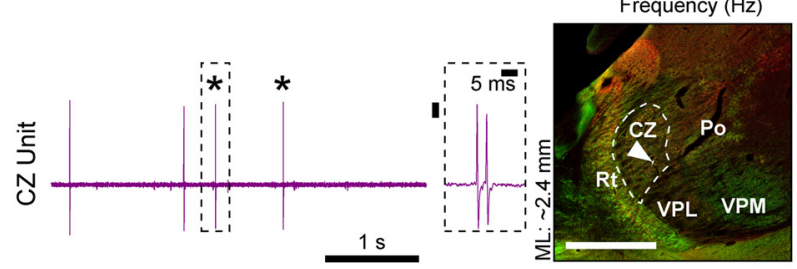

H
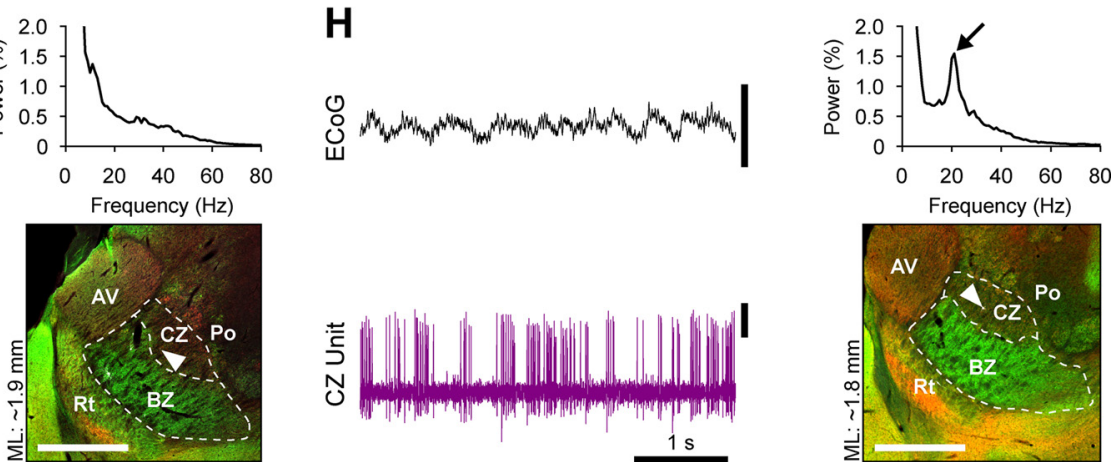

Figure 1. Brain state-dependent firing of identified neurons in the $\mathrm{BZ}$ and $\mathrm{CZ}$ of the motor thalamus in dopamine-intact and 6-OHDA-lesioned rats. A, Left, Spontaneous firing of a thalamic unit during cortical SWA in a dopamine-intact rat. Brain state was defined according to simultaneous ECoG recordings; during SWA, the ECoG was dominated by slow ( $\sim 1 \mathrm{~Hz})$ oscillations, as further verified in the ECOG power spectrum (top right). The thalamic unit fired stereotypical LTS bursts ${ }^{*}$ ) on most cycles of the cortical slow oscillation. Such bursts were characterized by 2-6 action potentials fired in rapid succession, often with a progressive attenuation of action potential amplitude during the burst (a typical burst is highlighted by the dashed box and also shown at higher temporal resolution in the inset). Right, Subsequent to electrophysiological recording with a glass electrode, the same neuron was juxtacellularly filled with neurobiotin, fluorescently labeled, and identified (white, arrowhead). The neuron was then localized to the BZ of motor thalamus, which was demarcated by intense GAD67 immunoreactivity (green) and sparse VGluT2 immunoreactivity (red) in the parasagittal tissue section ( $1.6 \mathrm{~mm}$ lateral of bregma [ML]). B, Spontaneous firing of an identified BZ neuron during SWA in a 6-OHDA-lesioned rat. C, Spontaneous firing of an identified BZ neuron recorded during cortical activation, as verified by a relative paucity of ECOG slow oscillations, in a dopamine-intact rat. The BZ neuron does not fire low-threshold spike bursts during cortical activation. $\boldsymbol{D}$, Spontaneous firing of an identified BZ neuron during cortical activation in a lesioned rat. Note the distinct peak at $\beta$ frequencies (black arrow) in the ECoG power spectrum. $\boldsymbol{E}$, Recording made during cortical SWA in a dopamine-intact rat of a neuron that was subsequently localized to the $C Z$ of motor thalamus, as delineated by sparse GAD67 and moderate VGluT2 immunoreactivities. $F$, Spontaneous firing of an identified CZ neuron during SWA in a lesioned rat. G, Spontaneous firing of an identified CZ 
Slow-Wave Activity BZ CZ
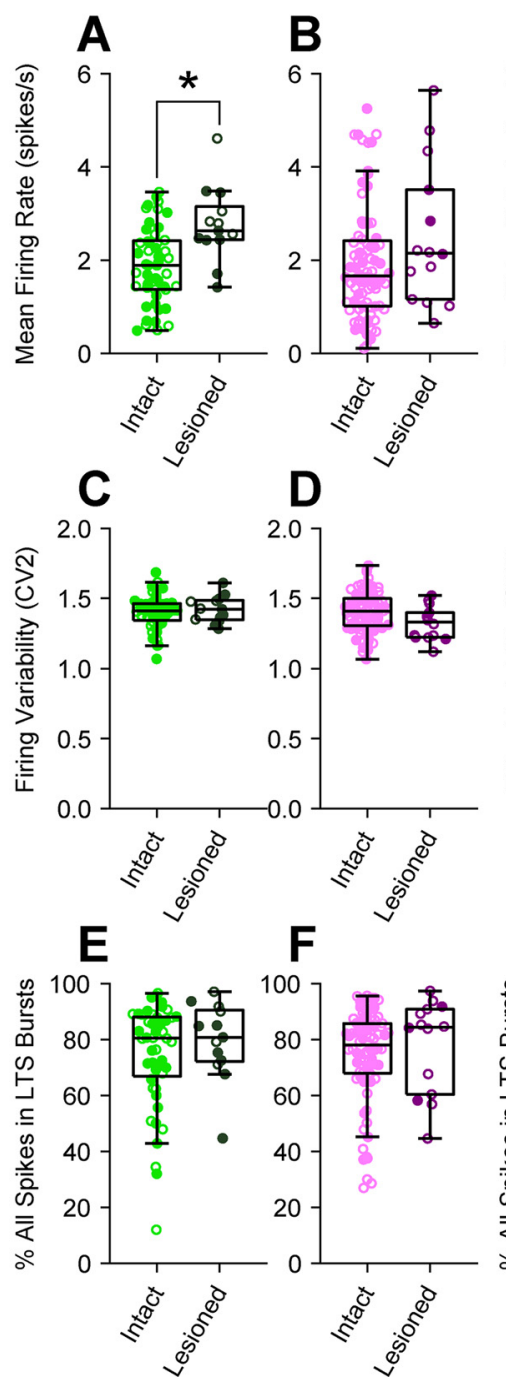

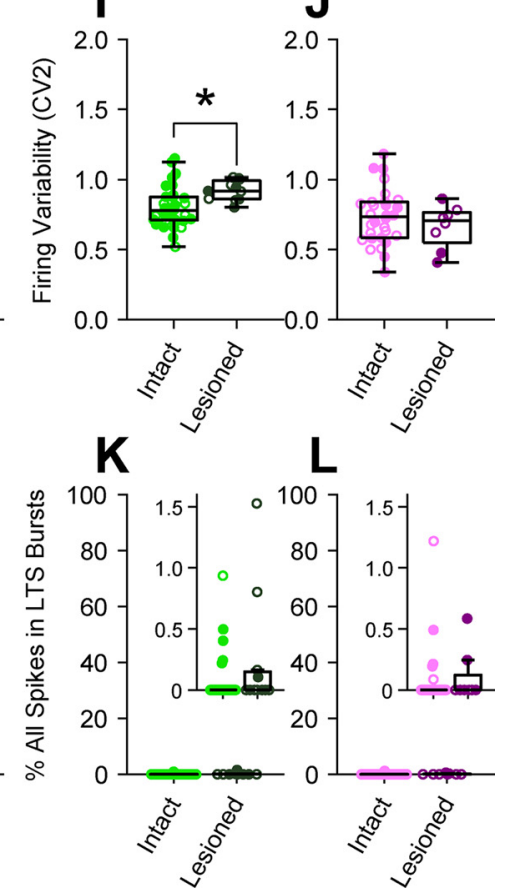

Cortical Activation

BZ CZ

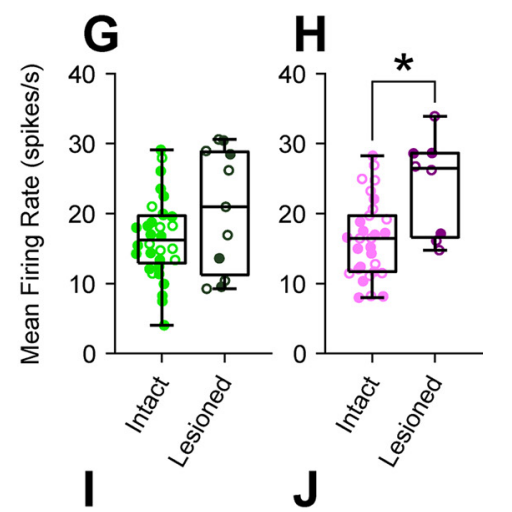

Figure 2. Quantitative comparisons of the firing rates and patterns of $B Z$ and $C Z$ neurons during two brain states in dopamine-intact and 6-OHDA-lesioned rats. Mean firing rates $(\boldsymbol{A}, \boldsymbol{B}, \mathbf{G}, \boldsymbol{H})$, firing variability, as indexed by the CV2 of ISIs $(\boldsymbol{C}, \boldsymbol{D}, \mathbf{I}$, $\boldsymbol{J})$, and mean percentage of all spikes occurring in LTS bursts $(\boldsymbol{E}, \boldsymbol{F}, \boldsymbol{K}, \boldsymbol{L})$ for all individual neurons recorded with glass electrodes in the BZ (light/dark greens) and in the CZ (pink/purple) of the motor thalamus. A-F, Thalamic activity parameters during SWA. Dopamine depletion was associated with an increase in the mean firing rates of BZ neurons (only), but did not alter the firing variabilities of either BZ or CZ neurons during SWA. G-L, Thalamic activity parameters during cortical activation. On average, the firing rates of $C Z$ neurons, but not $B Z$ neurons, were increased in lesioned rats. The activity patterns of motor thalamic neurons were intimately related to brain state; during cortical activation, their firing rates were relatively high and they rarely fired LTS bursts. Note the different $y$ axes in the insets in $\boldsymbol{K}$ and $\boldsymbol{L}$. Box plots in this and subsequent figures represent the nonoutlier values closest to the first and third quartiles (whiskers), interquartile range, and medians. Filled circles represent data from individual identified neurons. Open circles represent data from extrapolated neurons. ${ }^{*} p<0.05$ (exact $p$ values are in Results text), MWUTs.

$1 A, B$, insets). Many of these bursts satisfied the criteria for stereotypical LTS bursts (see Materials and Methods). Moreover, spikes were often fired in time with cortical slow $(\sim 1 \mathrm{~Hz})$ oscillations (Fig. $1 A, B)$. The activities of neurons in dopamine-intact and lesioned rats showed clear brain state dependency. Thus, during cortical activation, which was exemplified by a relative paucity of cortical slow oscillations (and, in the case of lesioned rats only, the emergence of exaggerated $\beta$ oscillations), the activity of identified BZ neurons in both dopamine-intact and lesioned rats was typified by relatively high mean firing rates ( $>10$ spikes/s) and a "tonic" irregular firing pattern (Fig. 1C,D). Neurons in BZ seldom fired LTS bursts during cortical activation. Importantly, the activity of identified CZ neurons was qualitatively similar to that of BZ neurons, regardless of brain state and whether recordings were made in dopamine-intact rats and or in lesioned rats (Fig. $1 E-H)$.

We next quantitatively assessed whether dopamine depletion altered the basic firing properties of BZ neurons or CZ neurons. For these analyses, we pooled together all identified and "extrapolated" neurons, that is, the unlabeled neurons whose locations could be accurately extrapolated from those of identified neurons (see Materials and Methods). We first considered thalamic activity recorded during cortical SWA (Fig. $2 A-F)$. On average, the firing rates of $\mathrm{BZ}$ neurons in lesioned rats $(2.76 \pm 0.22$ spikes/s [mean $\pm \mathrm{SEM}$ ]; $n=13$ neurons) were slightly, but significantly, higher $(U=488, p=0.002$, MWUT; Fig. 2A) than those of $\mathrm{BZ}$ neurons in dopamine-intact rats (1.88 \pm 0.06 spikes/s; $n=48$ neurons). In contrast, the firing rates of $\mathrm{CZ}$ neurons in lesioned rats $(2.51 \pm 0.41$ spikes/s; $n=14$ neurons) were similar ( $U=697, p=0.149$, MWUT; Fig. $2 B$ ) to those of CZ neurons in dopamine-intact rats $(1.93 \pm 0.09$ spikes/s; $n=80$ neurons). Dopamine depletion did not alter the firing variability (as indexed by CV2 measures) of either BZ neurons $(U=336, p=0.679$, MWUT; Fig. $2 C)$ or CZ neurons $(U=383, p=0.061$, MWUT; Fig. $2 D)$. During SWA, all motor thalamus neurons fired numerous LTS bursts (Fig. 1); on average, $\sim 75 \%$ of all spikes fired by BZ and CZ neurons during SWA occurred within LTS bursts (Fig. 2E, $F)$. Dopamine depletion did not alter the propensities of BZ and CZ neurons to fire spikes in LTS bursts $(U=354$ and 650, $p=0.465$ and 0.342 for $\mathrm{BZ}$ and $\mathrm{CZ}$ neurons, respectively, MWUT; Fig. 2E,F). We then considered thalamic activity recorded during cortical activation (Fig. 2G-L). On average, the firing rates of $\mathrm{BZ}$ neurons in lesioned rats $(20.48 \pm 2.66$ spikes/ s; $n=11$ neurons) were similar $(U=219, p=0.237$, MWUT; Fig. $2 G)$ to those of BZ neurons in dopamine-intact rats (16.49 \pm 0.52 spikes/s; $n=32$ neurons). In contrast, the firing neuron during cortical activation in a dopamine-intact rat. $\boldsymbol{H}$, Spontaneous firing of an identified $C Z$ neuron during cortical activation in a lesioned rat. In all parasagittal sections, rostral is toward the left, and dorsal is toward the top, with the borders of $B Z$ and $C Z$ indicated with dashed white lines. See also Extended Data Figure 1-1. AM, anteromedial thalamic nucleus; AV, anteroventral thalamic nucleus; MD, mediodorsal thalamic nucleus; Po, posterior nuclear group of thalamus; Rt, thalamic reticular nucleus; VPL, ventral posterolateral thalamic nucleus; VPM, ventral posteromedial thalamic nucleus. Scale bars: fluorescence images, 1 $\mathrm{mm}$. Vertical calibration bars: $0.5 \mathrm{mV}$. 
rates of $\mathrm{CZ}$ neurons in lesioned rats $(24.01 \pm 2.29$ spikes/s; $n=8$ neurons) were significantly higher $(U=190, p=0.013$, MWUT; Fig. 2H) than those of $\mathrm{CZ}$ neurons in control rats (16.39 \pm 0.70 spikes/s; $n=30$ neurons). Dopamine depletion increased the firing variability of $\mathrm{BZ}$ neurons $(U=275, p=0.006$, MWUT; Fig. 2I) but did not alter the firing variability of CZ neurons $(U=101, p=0.508$, MWUT; Fig. $2 J)$. During cortical activation, the proportions of all spikes included in LTS bursts were very low for neurons in both input zones $(0.23 \pm 0.15 \%$ and $0.07 \pm 0.02 \%$ for BZ neurons in lesioned and dopamine-intact rats, respectively; $0.10 \pm 0.08 \%$ and $0.07 \pm 0.03 \%$ for $\mathrm{CZ}$ neurons in lesioned and dopamine-intact rats; Fig. $2 K, L$ ). Dopamine depletion did not alter the propensities of BZ and CZ neurons to fire LTS bursts during cortical activation $(U=212$ and 132, $p=0.171$ and 0.543 for BZ and CZ neurons, respectively, MWUT; Fig. $2 K, L$ ).

In summary, these recordings of individual neurons accurately localized to one or the other input zone of the motor thalamus show that the firing rates of BZ neurons are not abnormally decreased after chronic dopamine depletion. These data also suggest that, when carefully controlling for two brain states, dopamine depletion does not increase the prevalence of LTS burst firing in either the $\mathrm{BZ}$ or $\mathrm{CZ}$.

\section{The firing of $\mathrm{BZ}$ neurons, but not $\mathrm{CZ}$ neurons, is aberrantly phase-locked to cortical slow oscillations after dopamine depletion}

Dopamine depletion can alter the patterning of BG neuron discharges during, and with respect to, the stereotyped cortical slow oscillation (Magill et al., 2001, 2006; Walters et al., 2007; Zold et al., 2012; Abdi et al., 2015). We addressed whether this also holds true for motor thalamus neurons. During SWA, the normalized power of ECoG slow oscillations (defined as $0.4-1.6 \mathrm{~Hz}$ ) (Nakamura et al., 2014; Sharott et al., 2017) simultaneously recorded with $\mathrm{BZ}$ neurons was on average similar between dopamine-intact rats and lesioned rats $(U=423, p=0.052$, MWUT; Fig. 3A). The power in ECoGs recorded with $\mathrm{CZ}$ neurons was also similar across animal groups $(U=570, p=0.920$, MWUT; Fig. 3A). The power of slow oscillations in single-unit activities in $\mathrm{BZ}$ was on average slightly higher in dopamine-intact rats than in lesioned rats $(U=432, p=0.035$, MWUT; Fig. $3 B)$, as was the coherence between ECoGs and BZ units at slow oscillation frequencies $(U=426, p=0.046$, MWUT; Fig. $3 C)$. The equivalent metrics in $\mathrm{CZ}$ were unaffected by dopamine depletion $(U=487$, $p=0.441$ for $\mathrm{CZ}$ units, MWUT, Fig. $3 B ; U=449, p=0.241$ for ECoG-CZ coherence, MWUT, Fig. $3 C$ ). These results suggest that dopamine depletion selectively alters the relationship between activities in frontal cortex and BZ. To test this further, we used the Hilbert transform to analyze the instantaneous phase of the spiking of thalamic neurons with respect to cortical slow oscillations at $0.4-1.6 \mathrm{~Hz}$ (Nakamura et al., 2014; Abdi et al., 2015; Garas et al., 2016; Sharott et al., 2017). On average, BZ neurons in dopamine-intact rats preferentially fired at the ascending phase of cortical slow oscillations (Fig. 3D), that is, at around the phase that cortical neurons transition from silence to coordinated firing (Sakata and Harris, 2009; Chauvette et al., 2010). This finely timed firing of BZ neurons was also evident in their individual phase histograms (Fig. 3E). Circular statistical analyses revealed that the spikes of all BZ neurons $(n=48)$ were significantly phase-locked to the slow oscillations $(p<0.05$, Rayleigh's Uniformity Test). Circular plots of the preferred phases of these phase-locked BZ neurons also demonstrated their strong tendency to fire at the ascending phase of the slow oscillations (Fig. $3 F)$; the mean angle of the preferred firing phases for the $\mathrm{BZ}$ neuron group was $283.4 \pm 3.8^{\circ}$ (Fig. $3 J$ ). Dopamine depletion profoundly disturbed the temporal coupling (phase locking) of $\mathrm{BZ}$ neuron firing to cortical slow oscillations (Fig. 3D-F,J). On average, and when compared with BZ neurons in dopamineintact rats, the peak of activity of $\mathrm{BZ}$ neurons in lesioned rats appeared smaller and broader in the linear phase histogram, indicating weaker and more variable phase locking as a population after dopamine depletion (Fig. 3D). Furthermore, most BZ neurons in lesioned rats preferentially fired at the descending phase of cortical slow oscillations (Fig. $3 D-F$ ), with a mean angle of firing of $31.7 \pm 15.4^{\circ}$ for the BZ neuron group (Fig. 3J). Accordingly, dopamine depletion resulted in a large $\left(\sim 100^{\circ}\right)$ and significant $(\mathrm{P}$ statistic $=11.40, p=0.001$, common median test) shift in the mean angles of firing of phase-locked BZ neurons. In stark contrast, dopamine depletion did not alter the temporal coupling of $\mathrm{CZ}$ neuron firing to cortical slow oscillations (Fig. $3 G-J)$. The similarities in the firing of CZ neurons in dopamineintact and lesioned rats were evident in linear phase histograms (Fig. $3 G, H$ ) and circular plots (Fig. 3I,J). Moreover, the mean angles of firing of phase-locked $\mathrm{CZ}$ neurons in control and lesioned rats were not shifted ( $\mathrm{P}$ statistic $=0.34, p=0.560$, common median test). When comparing vector lengths (Fig. $3 K$ ), which indicate how spiking activity of single neurons is concentrated around a given preferred phase, it was evident that BZ neurons in dopamine-intact rats had more consistent phaselocked firing than neurons in the other three groups, which were all similar $\left(H_{(3)}=36.35, p=6.30 \times 10^{-8}\right.$, Kruskal-Wallis ANOVA; $p=0.004$ for BZ intact vs BZ lesioned, $p=4.18 \times 10^{-8}$ for BZ intact vs $\mathrm{CZ}$ intact, $p=0.008$ for $\mathrm{BZ}$ intact vs $\mathrm{CZ}$ lesioned, $p=1.00$ for BZ lesioned vs CZ intact, $p=1.00$ for BZ lesioned vs CZ lesioned, $p=1.00$ for CZ intact vs CZ lesioned, post hoc Dunn's tests).

Together, these data show that the phase-locked firing of BZ neurons, but not $\mathrm{CZ}$ neurons, is impaired and inappropriately timed with respect to cortical slow oscillations after dopamine depletion. As such, these results collectively define one manifestation of an input zone-selective dysrhythmia in motor thalamus.

\section{The firing of SNr neurons is aberrantly phase-locked to cortical slow oscillations after dopamine depletion}

GABAergic SNr neurons innervate the BZ (Kuramoto et al., 2011), and their firing during SWA is altered by dopamine depletion (Belluscio et al., 2003; Tseng et al., 2005; Walters et al., 2007), together raising the possibility that the dysrhythmic firing of BZ neurons (Fig. 3) is mediated by SNr neurons. However, it is unclear whether the timing of $\mathrm{BZ}$ and $\mathrm{SNr}$ activity would support such a relationship. To address this, we used silicon probes to record single-unit activity in the $\mathrm{SNr}$ of dopamine-intact and 6-OHDA-lesioned rats during SWA (Fig. 4). Although the power of ECoG slow oscillations simultaneously recorded with $\mathrm{SNr}$ neurons was slightly lower in lesioned rats $(U=118, p=0.016$, MWUT; Fig. 4A), the power of slow oscillations in $\mathrm{SNr}$ unit activities was greatly elevated in lesioned rats $(U=143, p=3.14$ $\times 10^{-10}$, MWUT; Fig. 4B). Moreover, qualitative inspection of SNr unit activities (Fig. 4D), and analyses of phase histograms (Fig. 4E,F), suggested that the temporal coupling of SNr neuron firing to cortical slow oscillations was markedly stronger in lesioned rats. Consistent with this, the firing of $88.4 \%$ of $\mathrm{SNr}$ neurons (38 of 43) in lesioned rats was significantly phaselocked (Rayleigh's Uniformity Test) to cortical slow oscillations, whereas only $48.7 \%$ of SNr neurons (18 of 37) were significantly phase-locked in dopamine-intact rats (Fig. $4 J, K$ ). Moreover, the vector lengths of individual $\mathrm{SNr}$ neurons were longer in 

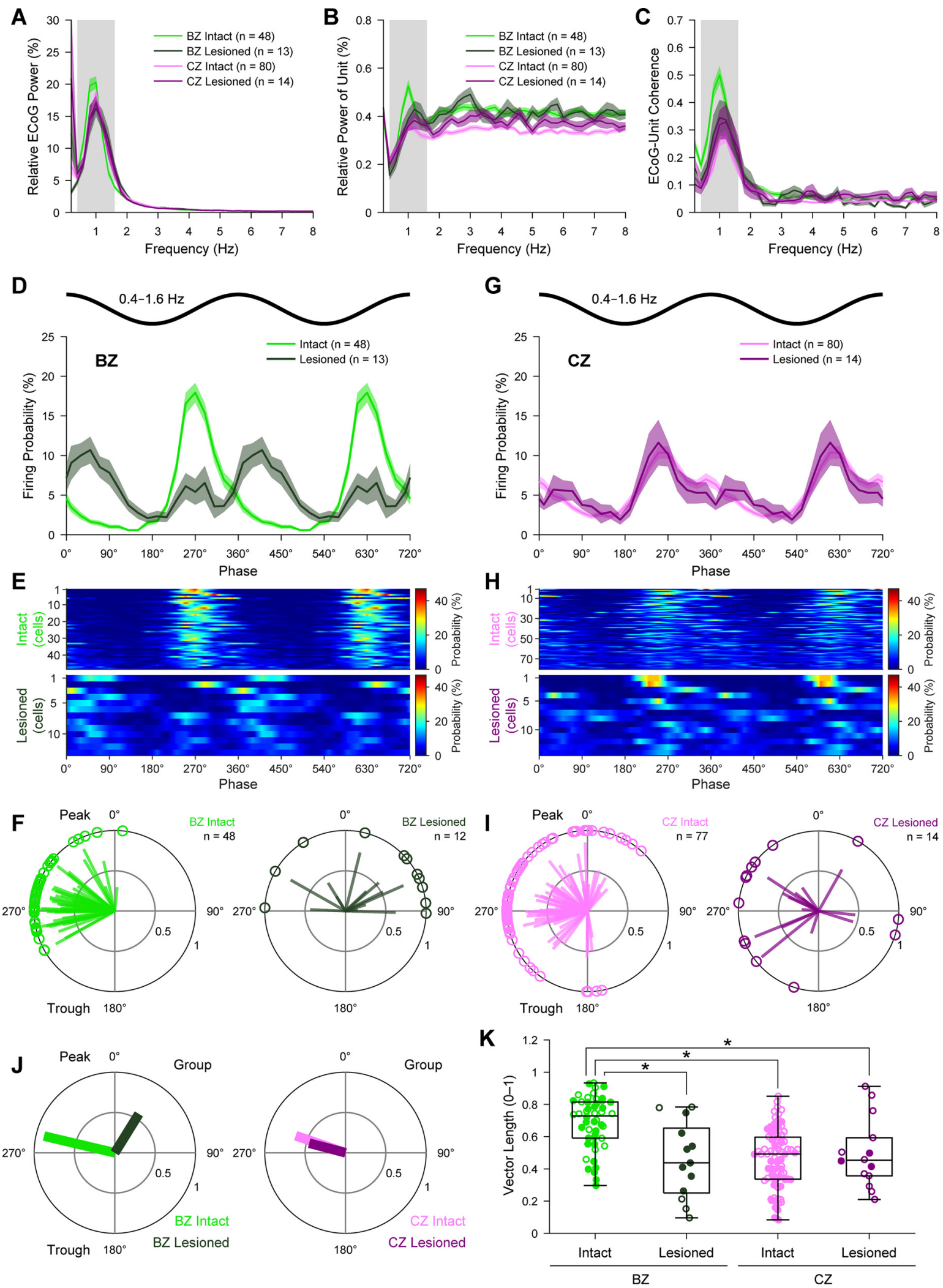

Figure 3. Spike timings of $B Z$ and $C Z$ neurons in relation to cortical slow oscillations. $A$, Mean power spectra of ECoGs simultaneously recorded with all $B Z$ neurons and all $C Z$ neurons in dopamine-intact and 6-0HDA-lesioned rats. Power is relative to that at $0.4-100 \mathrm{~Hz}$. Gray shading represents frequency band of slow oscillations analyzed $(0.4-1.6 \mathrm{~Hz})$. B, Mean power spectra of the spike discharges of all BZ neurons and CZ neurons. Note the peaks in power at frequencies similar to those of the cortical slow oscillations $(A)$. $C$, Mean coherence spectra between ECoGs and all BZ neurons and $C Z$ neurons (color coding and sample sizes as in $B$ ). $A-C$, Bin size of spectra is $0.2 \mathrm{~Hz}$. D, Linear phase histograms of the spike discharges of all BZ neurons with respect to cortical slow oscillations (bin size $=20^{\circ}$ ). Note the altered temporal coupling after dopamine depletion. For clarity, two cortical slow oscillation cycles are shown. $\boldsymbol{E}$, Heat map representation of linear phase histograms of individual $B Z$ neurons (bin size $=20^{\circ}$; neurons sorted by vector length, with longest at top). $F$, Circular plots of phase-locked firing of BZ neurons in dopamineintact and lesioned rats. In this and subsequent circular plots of individual neurons, only those neurons that qualified for analyses (i.e., $\geq 40$ spikes recorded) and that were also significantly phase-locked ( $p<0.05$, Rayleigh's Uniformity Test) are shown. Vectors of preferred firing of individual neurons are shown as lines radiating from the center. Greater vector lengths indicate 
lesioned rats $\left(U=375, p=5.06 \times 10^{-5}\right.$, MWUT; Fig. $\left.4 L\right)$, demonstrating that $\mathrm{SNr}$ neuron firing is more consistently phaselocked after dopamine depletion. These alterations in temporal coupling were not associated with changes in $\mathrm{SNr}$ neuron firing rates during SWA $(18.81 \pm 1.72$ and $21.12 \pm 0.21$ spike/s in lesioned and dopamine-intact rats, respectively; $U=620$, $p=0.091$, MWUT).

On average, $\mathrm{SNr}$ neurons in both dopamine-intact and lesioned rats tended to fire just after the peak of cortical slow oscillations (Fig. 4E,J,K); the mean angles of firing were $22.4 \pm 11.2^{\circ}$ and $28.1 \pm 9.1^{\circ}$, respectively, and they were similar ( $\mathrm{P}$ statistic $=0.45, p=0.501$, common median test; Fig. $4 K$ ). Given that BZ neurons in lesioned rats fired at a mean angle of $31.7 \pm 15.4^{\circ}$ (Fig. $3 J$ ), the average peak activity of $\mathrm{SNr}$ neurons would appear poorly timed to influence (inhibit) BZ neurons and shift their preferred phase of firing. However, we noted that some SNr neurons fired in a phasic "bursting" manner, rhythmically alternating between intense firing and near quiescence (Fig. $4 F)$, rather than quasi-sinusoidal fluctuations in firing. To investigate this, we first defined the bursts fired by a group of SNr neurons $(n=14)$, chosen for their high firing variability $(C V \geq 1.0)$, and analyzed the timing of their burst onsets and offsets with respect to cortical slow oscillations (Fig. 4G-I). Comparisons of linear phase histograms revealed that $\mathrm{SNr}$ burst onsets preferentially occurred just before the peak of cortical slow oscillations, and overlapped with reductions in BZ neuron activity, whereas $\mathrm{SNr}$ burst offsets preferentially occurred during the descending phase of cortical slow oscillations, and overlapped with the primary peak of BZ neuron activity (Fig. 4H,I). These correlations suggest that, after dopamine depletion, the emergence of aberrantly phase-locked burst firing in $\mathrm{SNr}$ is a valid candidate for mediating the phase shift and dysrhythmia of BZ neurons.

\section{The firing of individual $\mathrm{BZ}$ neurons, but not $\mathrm{CZ}$ neurons, is often aberrantly phase-locked to exaggerated cortical $\beta$ oscillations after dopamine depletion}

We next examined the extent to which the firing of individual neurons (identified and extrapolated) in motor thalamus is altered with respect to the cortical $\beta$-frequency $(15-30 \mathrm{~Hz})$ oscillations present during cortical activation (Fig. 5; also see Fig. $1 C$, $D, G, H)$. In line with previous reports (Mallet et al., 2008a, 2008b; Sharott et al., 2017), the ECoGs simultaneously recorded with thalamic neurons in 6-OHDA-lesioned rats showed significantly exaggerated $\beta$ oscillations compared with those recorded in dopamine-intact rats $\left(U=13, p=6.08 \times 10^{-6}\right.$ for ECoGs with $\mathrm{BZ}$ neurons, and $U=20, p=3.67 \times 10^{-4}$ for CZ neurons, MWUT; Fig. $5 A$; also see Fig. $1 D, H)$. The power spectra of spikes fired by BZ neurons, but not $\mathrm{CZ}$ neurons, displayed significantly enhanced and prominent $\beta$ oscillations after dopamine depletion $\left(U=29, p=4.55 \times 10^{-5}\right.$ for BZ neurons in lesioned vs dopamine-intact rats, and $U=150, p=0.291$ for CZ neurons, MWUT; Fig. 5B). Beta-band coherence between ECoGs and BZ neurons

\section{$\leftarrow$}

lower variance in the distribution around the mean phase angle. Each circle on the plot perimeter represents the preferred phase (i.e., mean phase of all the spikes) of an individual neuron. $\mathbf{G}-\mathbf{I}$, Same as in $\mathbf{D}-\boldsymbol{F}$, but for $\mathrm{CZ}$ neurons. $\boldsymbol{J}$, Mean vectors of the preferred firing phases of thalamic neurons in each group. Note the angular shift in the vector of BZ neurons in lesioned rats. $\boldsymbol{K}$, Vector lengths for all the spikes of all BZ and CZ neurons in dopamineintact and lesioned rats. Filled circles represent data from individual identified neurons. Open circles represent data from extrapolated neurons. ${ }^{*} p<0.05$ (exact $p$ values are in Results text), Dunn's tests following Kruskal-Wallis ANOVA. $\boldsymbol{A}-\boldsymbol{D}, \boldsymbol{G}$, Data are mean \pm SEM. $n$, the number of neurons/ECOGs analyzed. All individual neurons recorded with glass electrodes. was significantly augmented after dopamine depletion $(U=0$, $p=1.03 \times 10^{-6}$, MWUT; Fig. $\left.5 C\right)$, but this was not the case for CZ neurons $(U=67, p=0.060$, MWUT; Fig. $5 C)$. Phase histograms further suggested that the temporal coupling of BZ neuron firing to cortical $\beta$ oscillations was markedly stronger in lesioned rats (Fig. $5 D, E$ ). Consistent with this, the firing of all $\mathrm{BZ}$ neurons (11 of 11) in lesioned rats was significantly phase-locked (Rayleigh's Uniformity Test) to cortical $\beta$ oscillations, whereas only $6.3 \%$ of BZ neurons ( 2 of 32 ) were significantly phaselocked in dopamine-intact rats. Changes in the temporal coupling of $\mathrm{CZ}$ neurons to cortical $\beta$ oscillations were less marked (Fig. $5 F, G$ ), with minor proportions of $\mathrm{CZ}$ neurons exhibiting significantly phase-locked firing (3.3\% [ 1 of 30] and 37.5\% [3 of 8] of CZ neurons in dopamine-intact and lesioned rats, respectively). The disparities between $\mathrm{BZ}$ and $\mathrm{CZ}$ were unlikely to have arisen from systematic differences in cortical activity; the power of ECoG $\beta$ oscillations recorded with $\mathrm{BZ}$ or $\mathrm{CZ}$ neurons in lesioned rats was similar $(U=66, p=0.075$, MWUT). In lesioned rats, $\mathrm{BZ}$ neurons tended to discharge during the descending phase of cortical $\beta$ oscillations (Fig. 5D), with a mean angle of firing of $138.8 \pm 12.3^{\circ}$ for the group (Fig. $5 H$ ). When comparing the vector lengths of individual neurons (Fig. 5I), BZ neurons in lesioned rats had more consistent phase-locked firing than neurons in the other three groups, which were all similar $\left(H_{(3)}=\right.$ 28.72, $p=2.57 \times 10^{-6}$, Kruskal-Wallis ANOVA; $p=1.77 \times 10^{-6}$ for BZ intact vs BZ lesioned, $p=1.00$ for BZ intact vs $\mathrm{CZ}$ intact, $p=0.977$ for $\mathrm{BZ}$ intact vs $\mathrm{CZ}$ lesioned, $p=7.96 \times 10^{-6}$ for $\mathrm{BZ}$ lesioned vs $\mathrm{CZ}$ intact, $p=0.007$ for $\mathrm{BZ}$ lesioned vs $\mathrm{CZ}$ lesioned, $p=0.997$ for CZ intact vs CZ lesioned, post hoc Dunn's tests).

Together, these data show that individual BZ neurons, but not $\mathrm{CZ}$ neurons, tend to inappropriately engage with the exaggerated cortical $\beta$ oscillations that arise during activated brain states after dopamine depletion. As such, these results collectively reveal a second manifestation of an input zone-selective dysrhythmia in motor thalamus.

\section{Neuronal ensemble activity in the $\mathrm{BZ}$, but not $\mathrm{CZ}$, is aberrantly synchronized and phase-locked to exaggerated cortical $\boldsymbol{\beta}$ oscillations after dopamine depletion}

To gain insight into whether and how alterations in motor thalamus activity occurring after dopamine depletion extended to the collective outputs from larger ensembles of neurons, we analyzed silicon probe recordings of BUA that represents the spike firing of many neurons around the probe contacts (Moran et al., 2008; Moran and Bar-Gad, 2010). The BUA signals were extracted from wide-band recordings (Extended Data Fig. 6-1) and then used as continuous time series for spectral and circular statistical analyses. The use of DiI-coated linear probes with multiple recording contacts of known separation, together with post hoc anatomical verification of probe placement, allowed us to map the spatial profile of ensemble activity across discrete regions of $\mathrm{BZ}$ and CZ (Fig. 6A, $A^{\prime}$ ). Simultaneous recordings of BUA signals across the two thalamic input zones in lesioned rats showed that BZ ensemble activity, but not CZ ensemble activity, was often conspicuously modulated in time with ongoing cortical $\beta$ oscillations (Fig. 6B). Accordingly, some BUA signals in BZ, but not CZ, also exhibited prominent $\beta$ oscillations (Fig. $6 C$ ) and clear beta-band coherence with ECoGs (Fig. 6D). Of note, the pronounced temporal coupling, power, and coherence of BUA signals at $\beta$ frequencies were not always present across the whole $\mathrm{BZ}$, such that focal hot spots were instead evident (Fig. $6 B-D)$.

We extended the analysis of thalamic BUAs to quantitative comparisons across dopamine-intact and lesioned rats $(n=6$ and 

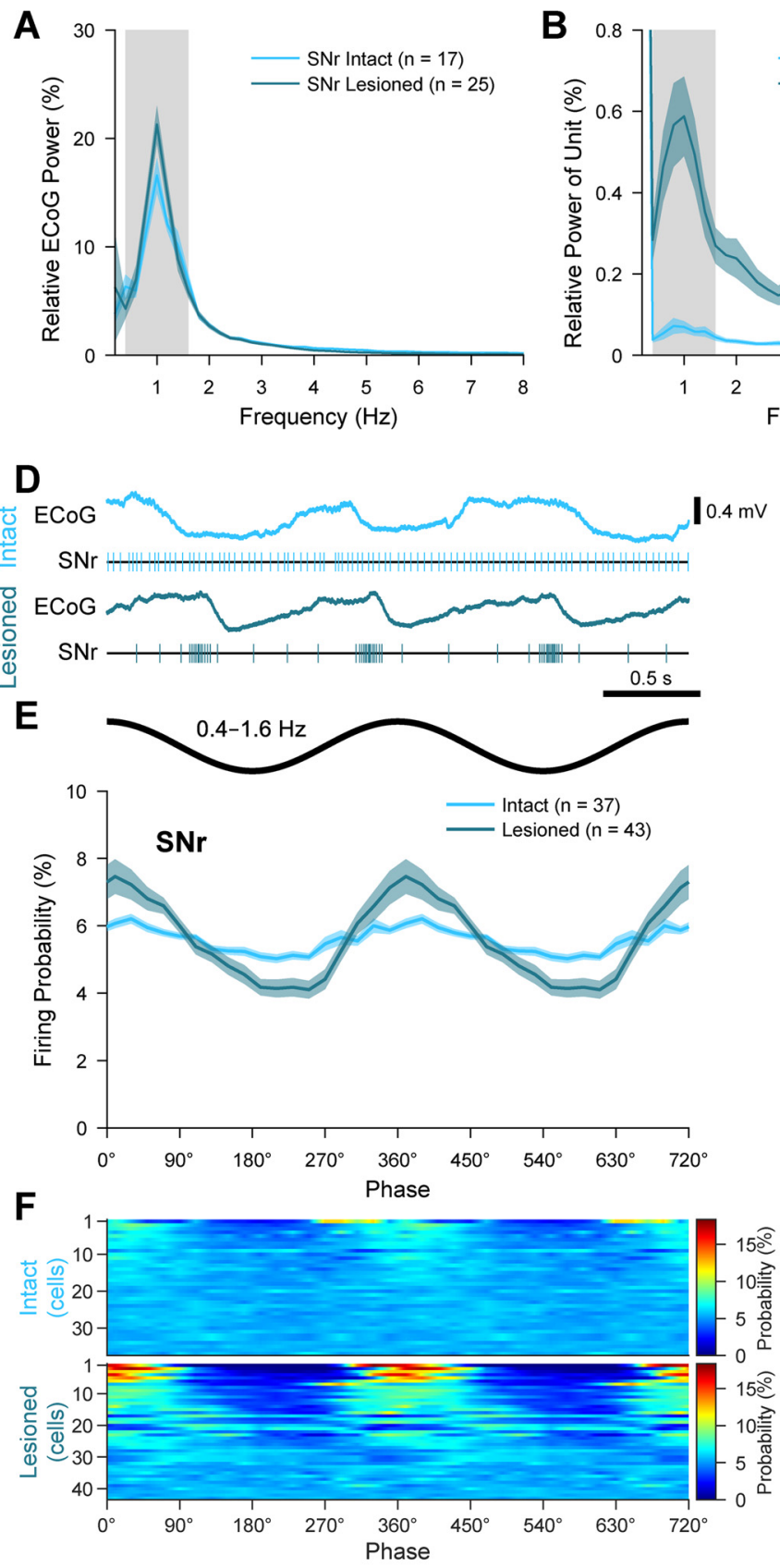

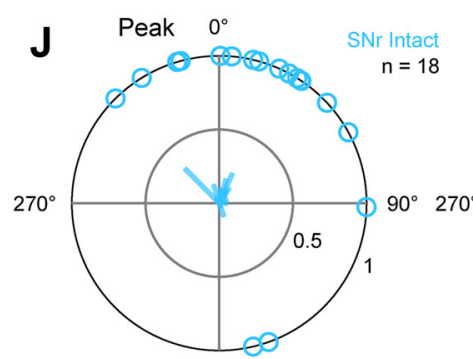

Trough $180^{\circ}$
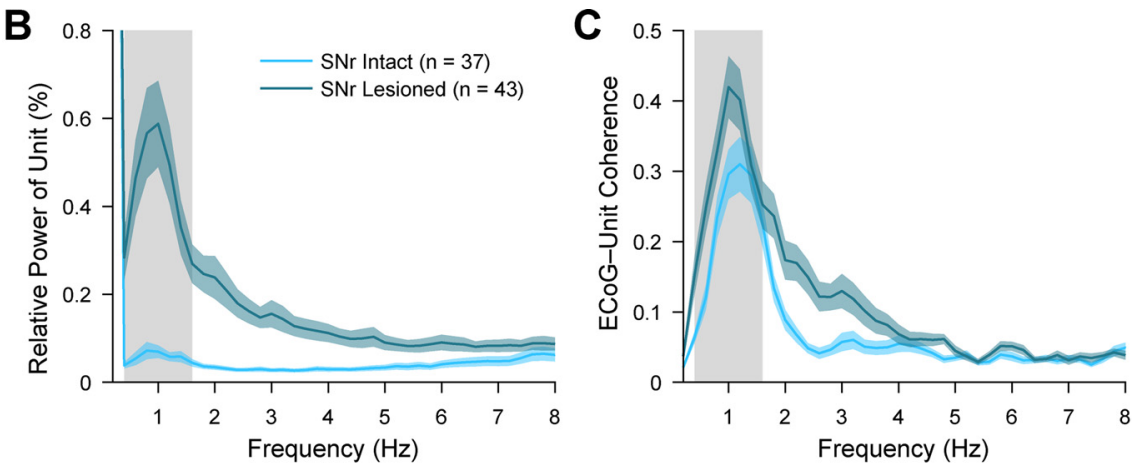

G

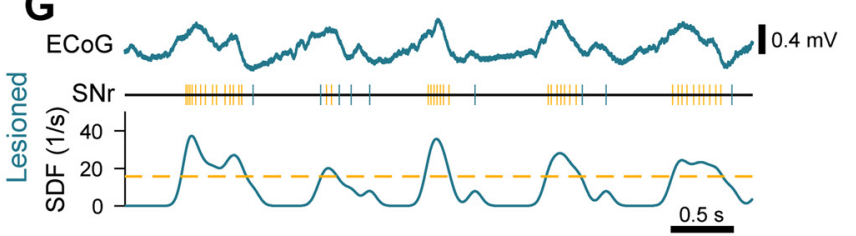

H
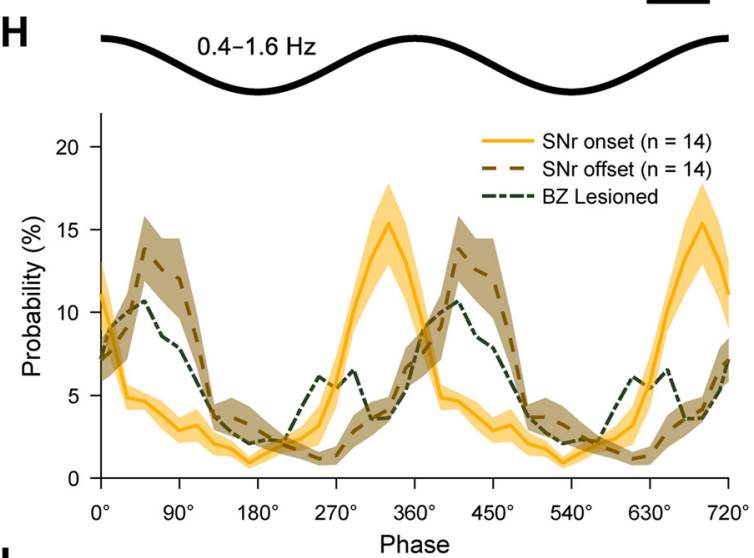

I
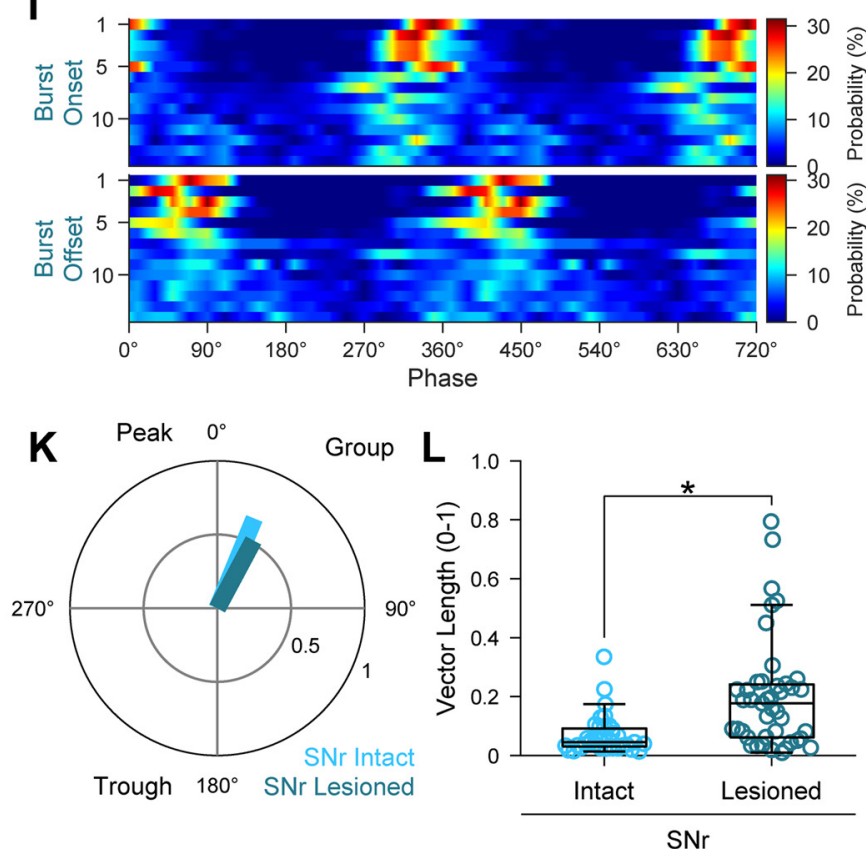

Figure 4. Spike timings of SNr neurons in relation to cortical slow oscillations. $A$, Mean power spectra of ECoGs simultaneously recorded with SNr neurons in dopamine-intact and 6-OHDAlesioned rats. Gray shading represents frequency band of slow oscillations analyzed $(0.4-1.6 \mathrm{~Hz})$. $\boldsymbol{B}$, Mean power spectra of the spike discharges of all SNr neurons. Note the peaks in power at frequencies similar to those of the cortical slow oscillations $(\boldsymbol{A})$. $\boldsymbol{C}$, Mean coherence spectra between ECoGs and all SNr neurons (color coding and sample sizes as in $\boldsymbol{B}$ ). $\boldsymbol{A}$-C, Bin size of spectra is $0.2 \mathrm{~Hz}$. D. Example spike trains of single SNr neurons, shown together with simultaneously recorded ECoGs for context, in an intact rat and a lesioned rat. $\boldsymbol{E}$, Linear phase histograms of the spike discharges of all SNr neurons with respect to cortical slow oscillations (bin size $=20^{\circ}$ ). Note the increased temporal coupling after dopamine depletion. $\boldsymbol{F}$, Heat map representation of linear phase histograms of individual SNr neurons (bin size $=20^{\circ}$; neurons sorted by vector length, with longest at top). $G$, Detection of bursts of spikes fired by $S \mathrm{Nr}$ neurons in lesioned rats. The 
7 rats, respectively) (Fig. 7). Among the power spectra of thalamic BUAs, only those recorded in the BZ of lesioned rats showed a conspicuous peak at $\beta$ frequencies (Fig. $7 B$ ). Accordingly, betaband power in BZ BUA signals was significantly increased after dopamine depletion $\left(U=7130, p=2.87 \times 10^{-8}, \quad\right.$ MWUT $)$. Similarly, the beta-band coherence between thalamic BUA signals and ECoGs was significantly increased for BZ, but not for CZ, after dopamine depletion $\left(U=5702, p=9.99 \times 10^{-14}\right.$ for $\mathrm{BZ}$, and $U=1126, p=0.444$ for CZ, MWUT; Fig. 7C). These disparities were unlikely to have arisen from systematic differences in cortical activity; the power of ECoG $\beta$ oscillations recorded with $\mathrm{BZ}$ or CZ BUA signals in lesioned rats was similar $(U=39, p=0.333$, MWUT; Fig. 7A). Beta-band coherence between pairs of BZ BUA signals was significantly increased after dopamine depletion $\left(U=127,009, p=2.08 \times 10^{-23}\right.$, MWUT; Fig. 7D). However, this was not the case for pairs of CZ BUA signals $(U=8024, p=0.901$, MWUT; Fig. 7E), nor for pairs made up of one BZ signal and one CZ signal $(U=34,599, p=0.834$, MWUT; Fig. $7 F)$. As noted above, not all BZ BUA signals showed clear peaks in power or coherence at $\beta$ frequencies after dopamine depletion (Fig. 6). Thus, to characterize the phase relationships between BZ BUA and cortical $\beta$ oscillations in lesioned rats, we first selected the BZ BUA signals with sample vector lengths longer than the 99.9th percentile of the sample vector lengths of the shuffled data (see Materials and Methods). When the amplitudes of these "significantly modulated" BUA signals $(n=35)$ were plotted with respect to cortical $\beta$ oscillations, it was evident that $\mathrm{BZ}$ ensemble activity tended to increase during the descending phase (Fig. $7 G, H$ ). On average, these BZ BUA signals showed a preferred mean angle of $123.4 \pm 7.2^{\circ}$ (Fig. 7I), which is similar ( $\mathrm{P}$ statistic $=1.08$, $p=0.300$, common median test) to the value we obtained for individual neurons in $\mathrm{BZ}\left(138.8 \pm 12.3^{\circ}\right.$; Fig. $\left.5 H\right)$. The power spectrum of the significantly modulated BZ BUA signals had a prominent peak in the beta band (Fig. $7 J$ ), as did their coherence spectrum with ECoGs (Fig. 7K).

In summary, these data collectively demonstrate that, after dopamine depletion, neuronal ensembles in the BZ, but not CZ, inappropriately synchronize their outputs at $\beta$ frequencies during cortical activation. Thus, input zone-selective dysrhythmia in motor thalamus also manifests at the level of population activity.

\section{Neuronal ensemble activity in the $\mathrm{SNr}$ is aberrantly synchronized and phase-locked to exaggerated cortical $\beta$ oscillations after dopamine depletion}

To provide further context for the dysrhythmic firing of BZ neurons in relation to exaggerated $\beta$ oscillations, we next tested

\footnotetext{
spike train of a single SNr neuron (middle) and its SDF (bottom) are shown together with the simultaneously recorded ECOG (top). Yellow dashed line in the SDF plot indicates the threshold (see Materials and Methods) for detecting the onsets and offsets of bursts. Yellow and blue ticks in the spike train represent spikes fired within and outside of bursts, respectively. $\boldsymbol{H}$, Linear phase histograms of the onsets (solid line in yellow) and offsets (dashed line in brown) of bursts fired by $\mathrm{SNr}$ neurons $\left(n=14\right.$; bin size $\left.=20^{\circ}\right)$ in lesioned rats compared with the firing of BZ neurons in lesioned rats (dot and dashed line in gray). $I$, Heat map representation of linear phase histograms of burst onsets and burst offsets of the $14 \mathrm{SNr}$ neurons (bin size $=20^{\circ}$; neurons sorted by vector length). $J$, Circular plots of phase-locked firing of individual $\mathrm{SNr}$ neurons in dopamine-intact and lesioned rats. $\boldsymbol{K}$, Mean vectors of the preferred phases of $S \mathrm{Nr}$ neurons in each group. $L$, Vector lengths for all the spikes of all $S \mathrm{Nr}$ neurons in dopamine-intact and lesioned rats. ${ }^{*} p=5.06 \times 10^{-5}$, MWUT. $\boldsymbol{A}$-C, $\boldsymbol{E}, \boldsymbol{H}$, Data are mean \pm SEM. $n$, the number of neurons/ECoGs analyzed. All neurons are single units recorded with silicon probes.
}

$\leftarrow$ whether neuronal ensemble activity in $\mathrm{SNr}$ during cortical activation was similarly altered by dopamine depletion (Figs. 8 and 9). Following the experimental approach used to investigate motor thalamus (Figs. 6 and 7), the use of DiI-coated silicon probes, together with post hoc anatomical verification of probe placement, allowed us to map the spatial profile of activity across discrete regions of $\mathrm{SNr}$ (Fig. $8 A, A^{\prime}$ ). Simultaneous recordings of BUA signals at multiple sites within the $\mathrm{SNr}$ of lesioned rats showed that ensemble activity was often conspicuously modulated in time with ongoing cortical $\beta$ oscillations (Fig. $8 B$ ). Some SNr BUA signals also exhibited prominent $\beta$ oscillations (Fig. $8 C$ ) and clear beta-band coherence with ECoGs (Fig. 8D). The pronounced temporal coupling, power, and coherence of BUA signals at $\beta$ frequencies typically manifested as focal hot spots (Fig. 8B-D).

Quantitative comparisons of SNr BUA signals recorded in dopamine-intact and lesioned rats ( $n=5$ and 5 rats, respectively) revealed several important differences (Fig. 9). The power spectrum of SNr BUA signals in lesioned rats, but not dopamineintact rats, showed a prominent peak at $\beta$ frequencies (Fig. 9B). Accordingly, beta-band power in SNr BUA signals was significantly increased after dopamine depletion $(U=5098, p=8.19$ $\times 10^{-9}$, MWUT). The beta-band coherence between SNr BUA signals and ECoGs significantly increased after dopamine depletion $\left(U=1494, p=5.41 \times 10^{-31}\right.$, MWUT; Fig. 9C), as did the beta-band coherence between pairs of $\mathrm{SNr}$ BUA signals $\left(U=77,056, p=3.97 \times 10^{-50}\right.$, MWUT; Fig. $\left.9 D\right)$. Because not all SNr BUA signals showed clear peaks in power or coherence at $\beta$ frequencies after dopamine depletion (Fig. 8), we again selected a set of significantly modulated SNr BUA signals $(n=92)$ for analyses of phase relationships between $\mathrm{SNr}$ ensemble activity and cortical $\beta$ oscillations in lesioned rats (Fig. 9G-I). Ensemble activity in $\mathrm{SNr}$ tended to increase during the ascending phase of cortical $\beta$ oscillations (Fig. 9G,H), with, on average, a preferred mean angle of $266.1 \pm 2.3^{\circ}$ (Fig. 9I). The power spectrum of the significantly modulated SNr BUA signals had a prominent peak in the beta band (Fig. 9J), as did their coherence spectrum with ECoGs (Fig. 9K). On comparing the rhythmic modulation (preferred phases) of SNr BUA signals (Fig. 9H,I) and BZ BUA signals (Fig. 7H,I), an "antiphase" relationship was apparent, such that peaks in $\mathrm{SNr}$ ensemble activity were approximately timed with troughs in $\mathrm{BZ}$ ensemble activity, and vice versa. We also isolated single units from the same probe recordings in SNr. Beta-band coherence between SNr single units and ECoGs was significantly augmented after dopamine depletion $\left(U=173, p=6.03 \times 10^{-6}\right.$, MWUT; Fig. 9E). The firing of $52 \%$ of SNr single units (24 of 46) in lesioned rats was significantly phase-locked (Rayleigh's Uniformity Test) to cortical $\beta$ oscillations, whereas none of the $\mathrm{SNr}$ single units ( 0 of 23) in dopamine-intact rats were similarly phase-locked. On average, the significantly phase-locked $\mathrm{SNr}$ single units in lesioned rats fired at a preferred mean angle of $221.9 \pm 11.1^{\circ}$ (Fig. $9 F$ ). These alterations in temporal coupling were not associated with changes in $\mathrm{SNr}$ neuron firing rates during cortical activation $(29.25 \pm 2.06$ and $28.08 \pm 0.32 \mathrm{spike} / \mathrm{s}$ in lesioned and dopamine-intact rats, respectively; $U=556, p=0.736$, MWUT).

In summary, these data demonstrate that, after dopamine depletion, neuronal ensembles in the $\mathrm{SNr}$ inappropriately synchronize their outputs at $\beta$ frequencies during cortical activation. They further suggest that aberrantly phase-locked firing of $\mathrm{SNr}$ neurons is a valid candidate for mediating the related dysrhythmia of BZ neurons. 


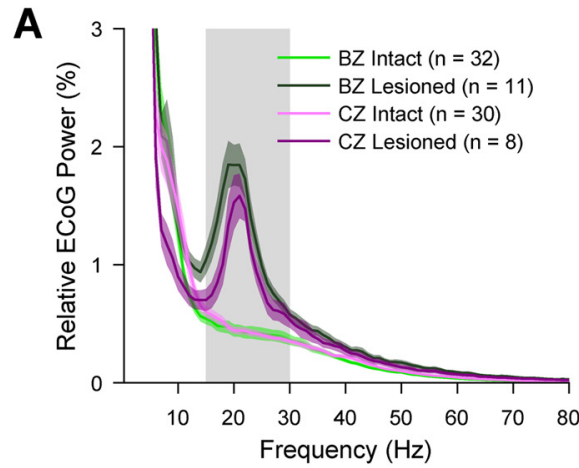

D

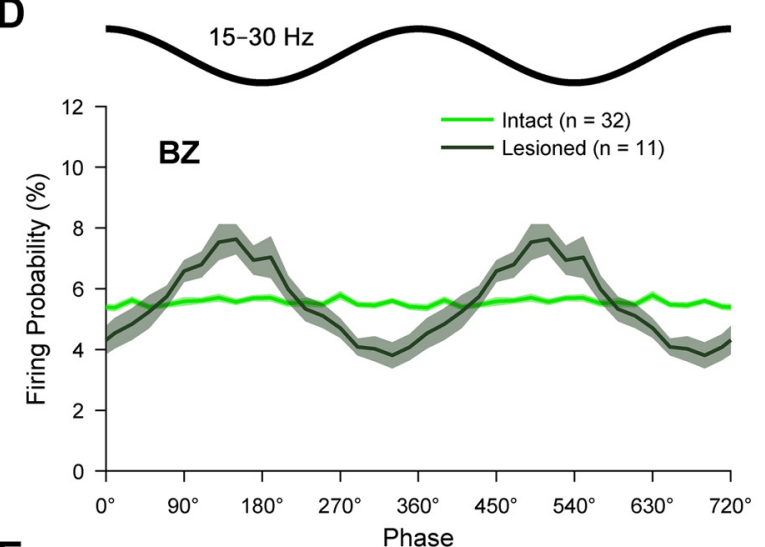

E
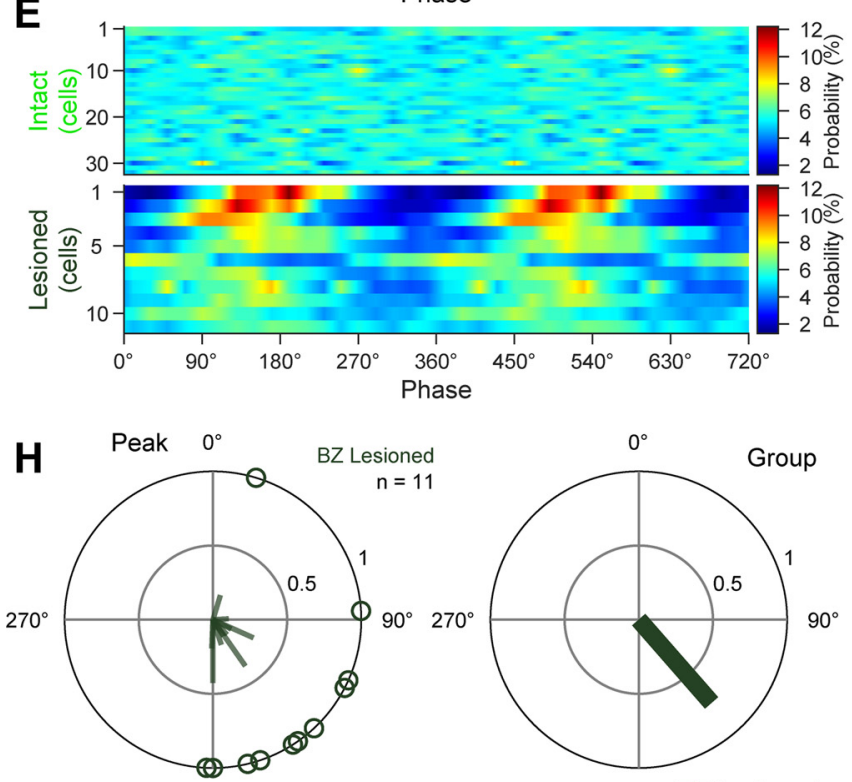

Trough $180^{\circ}$
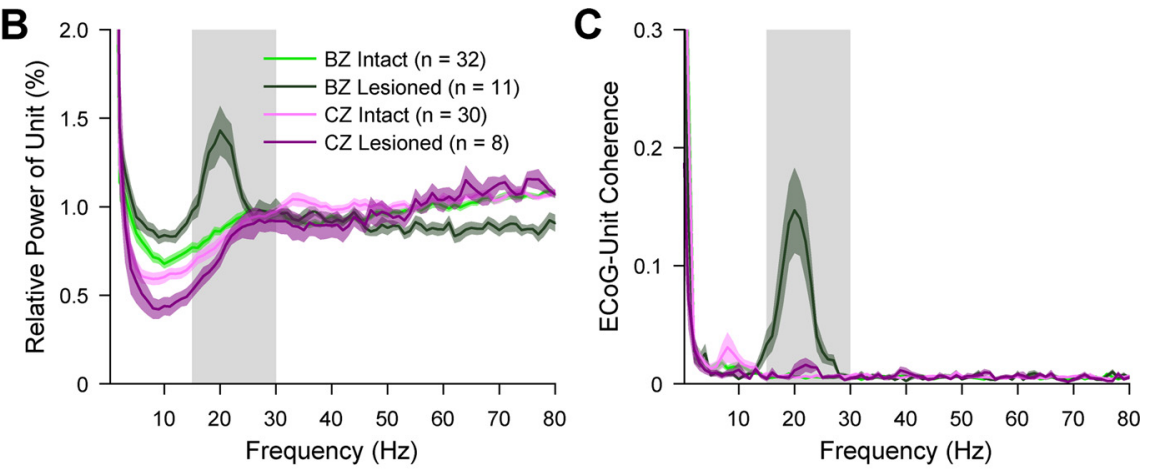

$\mathbf{F}$

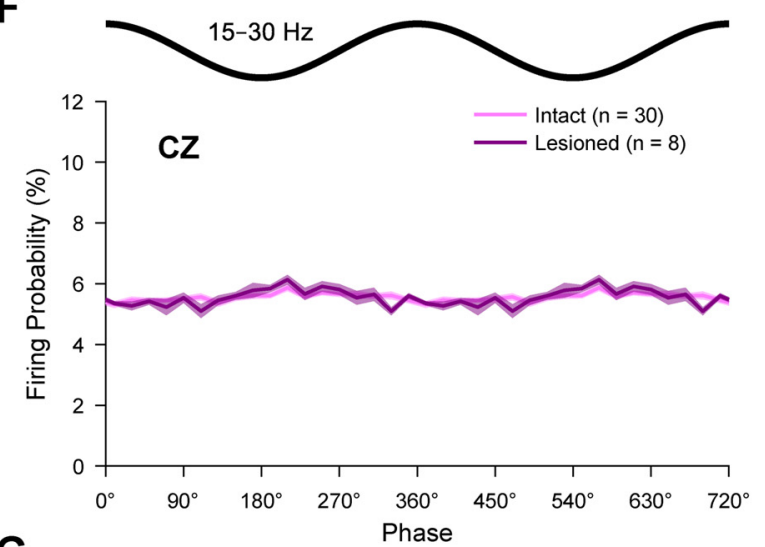

$\mathbf{G}$
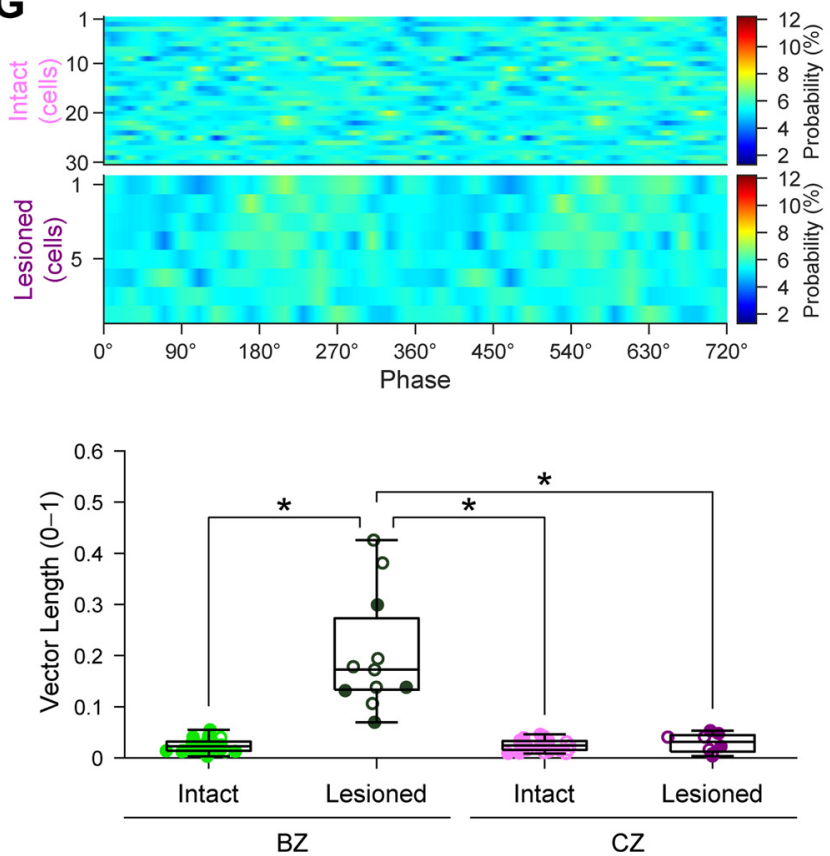

Figure 5. Spike timings of $B Z$ and $C Z$ neurons in relation to cortical $\beta$ oscillations. $A$, Mean power spectra of ECoGs simultaneously recorded with all $B Z$ neurons and all $C Z$ neurons in dopamine-intact and 6-0HDA-lesioned rats. Power is relative to that at 1-100 Hz. Gray shading represents frequency band of $\beta$ oscillations analyzed (15-30 Hz). $\boldsymbol{B}, \mathrm{Mean}$ power spectra of the spike discharges of $\mathrm{BZ}$ neurons and $\mathrm{CZ}$ neurons. Note the peak in power at $\beta$ frequencies for BZ neurons in lesioned rats; their peak frequency was similar to that of cortical $\beta$ oscillations $(\boldsymbol{A})$. $C$, Mean coherence spectra between ECOGs and all BZ neurons and CZ neurons (color coding and sample sizes as in $\boldsymbol{B}$ ). Note the peak in coherence at $\beta$ frequencies for BZ neurons in lesioned rats. $A$-C, Bin size of spectra is $1 \mathrm{~Hz}$. D, Linear phase histograms of the spike discharges of all BZ neurons with respect to cortical $\beta$ oscillations (bin size $=20^{\circ}$ ). Note the increased temporal coupling after dopamine depletion. For clarity, two cortical beta oscillation cycles are shown. $\boldsymbol{E}$, Heat map representation of linear phase histograms of individual $B Z$ neurons (bin size $=20^{\circ}$; neurons sorted by vector length, with longest at top). $\boldsymbol{F}, \boldsymbol{G}$, Same as in $\boldsymbol{D}, \boldsymbol{E}$, but for $(Z$ neurons. $\boldsymbol{H}$, Left, Circular plot of the phase-locked firing of individual $B Z$ neurons in lesioned rats. Right, Mean vector of the preferred phases of $B Z$ neurons in lesioned rats. $I$, Vector lengths for all the spikes of all BZ and $C Z$ neurons in dopamine-intact and lesioned rats. Filled circles represent data from individual identified neurons. Open circles represent data from extrapolated neurons. ${ }^{*} p<0.05$ (exact $p$ values are in Results text), Dunn's tests following Kruskal-Wallis ANOVA. $\boldsymbol{A}$ $D, F$, Data are mean \pm SEM. $n$, the number of neurons/ECoGs analyzed. All individual neurons recorded with glass electrodes. 

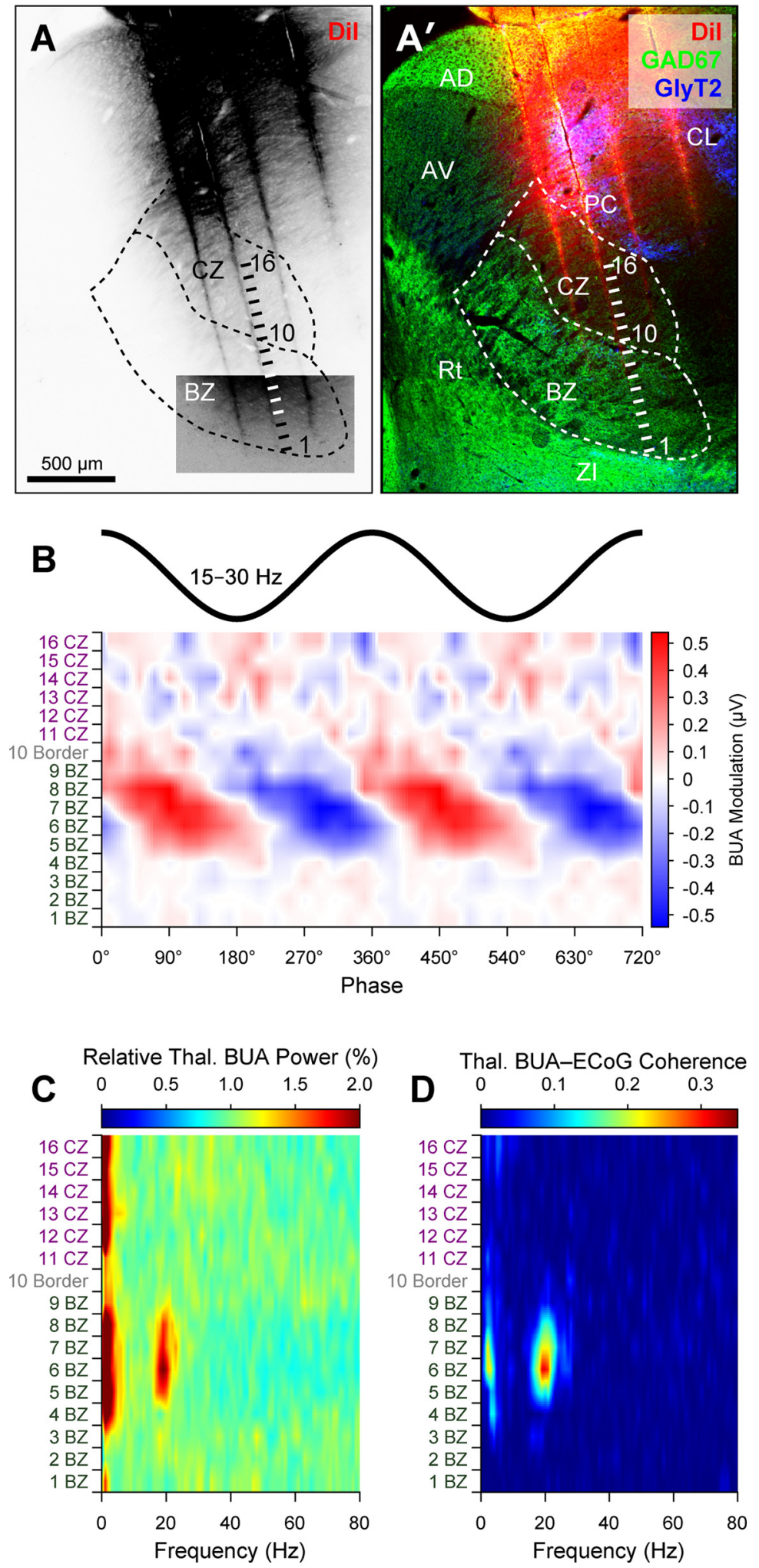

Figure 6. Example localization of BUA signals in the motor thalamus, and their relationship with cortical $\beta$ oscillations. $\boldsymbol{A}$, Image of a parasagittal tissue section from a 6-0HDA-lesioned rat, with Dil fluorescence signal (in an inverted tone for clarity) marking four penetration tracks made at different times by a Dil-coated silicon probe during recordings in vivo. An overlaid image with enhanced contrast reveals the ends of three tracks. Short lines indicate the estimated positions of the probe's 16 recording contacts along one such track. $\boldsymbol{A}^{\prime}$, The Dil signal (red) was localized with respect to the BZ and $C Z$ of the motor thalamus, as delineated by GAD67 (green) and GlyT2 (blue) immunofluorescence, on the same section as in $\boldsymbol{A}$. Probe contacts 1-9 were considered to have been within BZ, whereas contacts 11-16 were in CZ. B, BUA signals (also see Extended Data Fig. 6-1) from the 16 recording contacts at the positions indicated in $\boldsymbol{A} / \boldsymbol{A}^{\prime}$, averaged according to the instantaneous phases of cortical $\beta$ oscillations (bin size $=20^{\circ}$ ) and plotted as a heat map. Note the

\section{GABA infusions into motor thalamus reduce Parkinsonian $\beta$ oscillations in motor cortex}

Excessively synchronized $\beta$-frequency output from the BZ (Figs. 5-7) might be causally important for the expression of abnormal $\beta$ oscillations in the BZ's principal target, namely, the motor cortex. We reasoned that, if this were the case, then suppressing BZ neuron activity would reduce the power of abnormal $\beta$ oscillations in motor cortex. To test this, we recorded ECoG $\beta$ oscillations during cortical activation in lesioned rats ( $n=10)$ before, during, and after infusions of a small volume $(60 \mathrm{nl})$ of GABA solution $(0.5 \mathrm{M})$ via a glass pipette inserted into the motor thalamus (Fig. 10). We chose to microinfuse GABA (rather than other agonists of GABA receptors) because the inactivation effects of similarly small infusions of GABA typically abate within a few minutes (Kojima and Doupe, 2009); this in turn allowed us to perform repeated infusions (using a minimum interval of $10 \mathrm{~min}$ ) at one or more thalamic sites in a single animal (Fig. 10A,B). Using this approach, we thus aimed to rapidly, but transiently, quash or otherwise perturb neuronal activity in a small volume of thalamic tissue. By marking the trajectories of the same glass micropipettes with synthetic fluorescent markers, we were able to accurately localize the GABA infusion sites to the $\mathrm{BZ}$ or $\mathrm{CZ}$ (Fig. 10C). Our approach is to be contrasted with that used in a previous study (Brazhnik et al., 2016), where much larger volumes $(\sim 10 \times)$ of the longer-lasting $\mathrm{GABA}_{\mathrm{A}}$ receptor agonist muscimol were injected via much larger cannulas $(>10 \times$ in diameter) into the ventral medial (VM) nucleus and thereabouts, with the effects of drug injection being quantified hours after the event.

Brief microinfusions (24.4 $\pm 1.0 \mathrm{~s})$ of GABA at sites post hoc localized to BZ caused rapid (within $\sim 25 \mathrm{~s}$ of infusion onset) and reproducible decreases in the power of cortical $\beta$ oscillations (Fig. $10 A-C$ ). On average, the nadir in cortical beta power occurred at $\sim 100 \mathrm{~s}$ from the onset of GABA infusions at

large-amplitude phasic modulation of BUA signals at contacts 5 9 within the BZ. C, Power spectra of BUA signals, from the same recordings as in $\boldsymbol{B}$, shown as a heat map. Power is relative to that at $1-100 \mathrm{~Hz}$. D, Spectra of coherence between the same BUA signals and the simultaneously recorded ECOG. Note the focal nature of thalamic power and coherence at $\beta$ frequencies. $\boldsymbol{C}, \boldsymbol{D}$, Bin size of spectra is $1 \mathrm{~Hz}$. In the parasagittal section shown in $\boldsymbol{A} / \boldsymbol{A}^{\prime}$, rostral is toward the left, and dorsal is toward the top. $A D$, anterodorsal thalamic nucleus; $C L$, central lateral thalamic nucleus; PC, paracentral thalamic nucleus; Rt, thalamic reticular nucleus; ZI, zona incerta. For abbreviations of other thalamic nuclei, see Figure 1. 

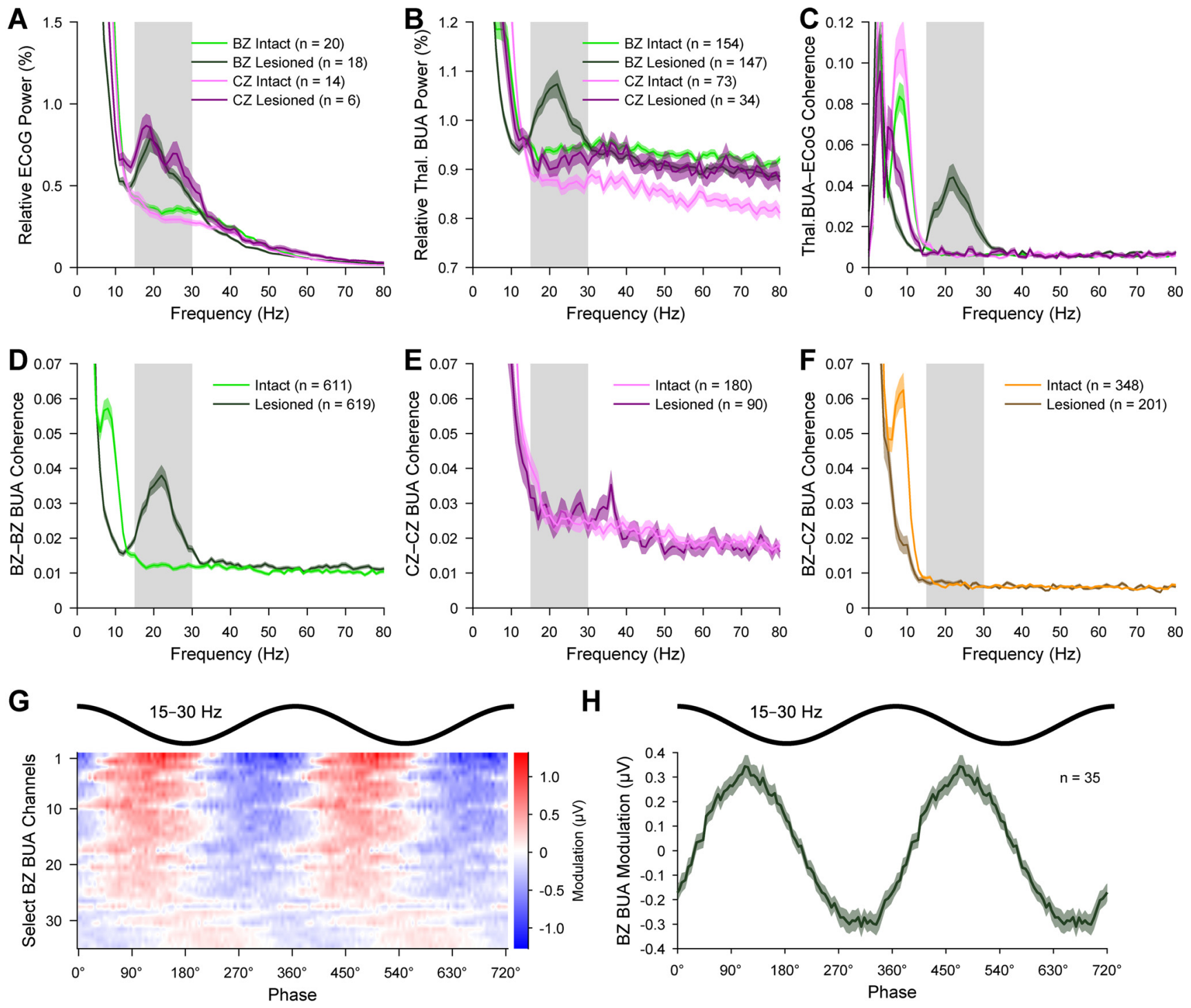

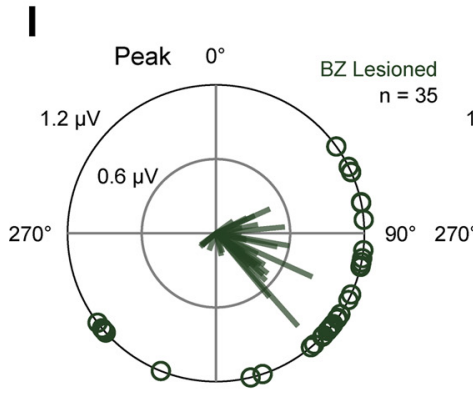

Trough $180^{\circ}$

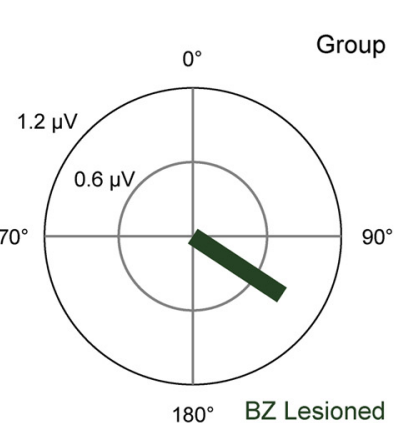

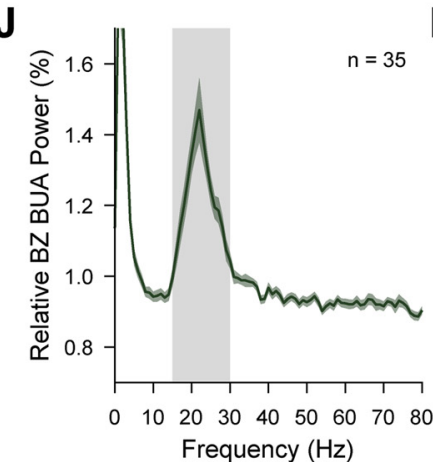

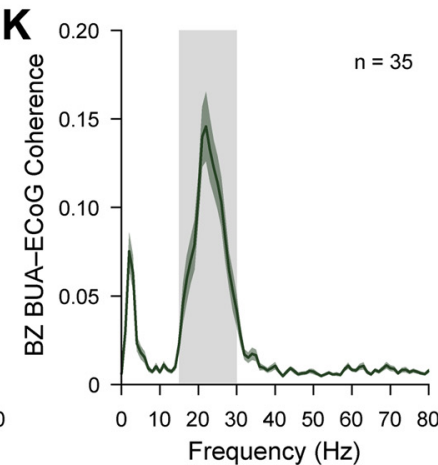

Figure 7. BUA signals in the motor thalamus during cortical activation in dopamine-intact and 6-OHDA-lesioned rats. $A$, Mean power spectra of ECoGs simultaneously recorded with BUA in BZ and CZ in dopamine-intact and 6-0HDA-lesioned rats. Gray shading represents frequency band of $\beta$ oscillations analyzed (15-30 Hz). $\boldsymbol{B}$, Mean power spectra of BUA signals recorded in motor thalamus. Note the peak in power at $\beta$ frequencies for the BZ BUA signals in lesioned rats. $C$, Mean coherence spectra between the thalamic BUA signals and ECoGs (color coding and sample sizes as in $B$ ). Note the peak in coherence at $\beta$ frequencies for BZ BUA signals in lesioned rats. $\boldsymbol{D}$, Coherence spectra of simultaneously recorded pairs of BZ BUA signals. $\boldsymbol{E}$, Same as in $\boldsymbol{D}$, but for pairs of $C Z$ BUA signals. $\boldsymbol{F}$, Coherence spectra between simultaneously recorded pairs of BZ and CZ BUA signals. $\mathbf{G}$, Heat map representation of the phase-averaged waveforms of the significantly modulated BZ BUA signals ( $n=35$ ) in lesioned rats (bin size $=5^{\circ}$; BUA signals sorted by vector length). $\boldsymbol{H}$, Group average of waveforms shown in $\boldsymbol{G}$. Positive modulations of BZ BUA tended to occur during the descending phase of cortical $\beta$ oscillations. $\boldsymbol{I}$, Left, Circular plots of the individual significantly modulated BZ BUA signals in lesioned rats. Vectors representing the phase preference of individual BUA signals are shown as lines radiating from the center. Greater vector lengths indicate greater modulation of BUA amplitude around the mean phase angle. Each circle on the plot perimeter represents the preferred phase of an individual BUA signal. Right, Mean vector of the preferred phases of all significantly modulated BZ BUA signals. J, Mean power spectrum of the significantly modulated BZ BUA signals. $\boldsymbol{K}$, Mean coherence spectrum between significantly modulated BZ BUA signals and the simultaneously recorded ECoGs. $\boldsymbol{A}-\boldsymbol{F}, \boldsymbol{H}, \boldsymbol{J}, \boldsymbol{K}$, Data are mean \pm SEM. Bin size of power and coherence spectra is $1 \mathrm{~Hz}$. $n$, the number of individual ECoG recordings $(\boldsymbol{A})$, the number of individual $B U A$ signals recorded $(B, C$, the number of pairs of BUA signals recorded $(\boldsymbol{D}-\boldsymbol{F})$, and the number of the significantly modulated BZ BUA signals $(\boldsymbol{H}-\boldsymbol{K})$. All BUA recorded with silicon probes. 
BZ sites $(n=26)$, after which beta power steadily increased (Fig. 10D). Effect size, defined as the percentage reduction in cortical beta power at 50 $150 \mathrm{~s}$ after GABA infusion compared with power during the $100 \mathrm{~s}$ immediately before infusion, was on average $\sim 50 \%$ for infusions at BZ sites (Fig. $10 D-F)$. That said, effect sizes across different BZ infusions sites and all animals were highly variable (Fig. $10 D-F)$, which might relate to the focal hot spot expression of $\beta$ oscillations in the BZ (see Fig. 6). Infusion of GABA at CZ sites $(n=13)$ also led to reductions in cortical beta power, with an average time course of effect that was akin to that after infusions at BZ sites (Fig. 10D). However, compared with infusions at BZ sites, the effect sizes of infusions at CZ sites were on average significantly smaller $(U=213, p=0.038$, MWUT; Fig. 10E). As a further distinction, effects sizes of $>60 \%$ were limited to GABA infusions made at BZ sites (Fig. 10E,F).

Together, these pharmacological perturbation analyses suggest that ongoing activity in the motor thalamus is permissive for exaggerated $\beta$ oscillations in motor cortex, with output from the BZ being of special importance for the maintenance of these pathological cortical rhythms.

\section{Discussion}

Here, we elucidate how chronic dopamine depletion alters the temporal dynamics of electrical activity within the two major input zones of motor thalamus in vivo. Our data demonstrate that distinct, brain state-dependent manifestations of dysrhythmia selectively emerge within the $\mathrm{BZ}$ in Parkinsonism.

\section{Neuronal firing rates in \\ Parkinsonism}

The direct/indirect pathways model predicts that motor thalamus neurons are hypoactive in Parkinsonism (DeLong, 1990; Smith et al., 1998; Galvan et al., 2015). Our recordings of individual neurons accurately localized to the $\mathrm{BZ}$ and $\mathrm{CZ}$ do not support this prediction. Previous work in anesthetized rats shows that dopamine depletion only alters the firing rates of some $B G$ neurons in the expected manner during certain brain states (Belluscio et al., 2003;
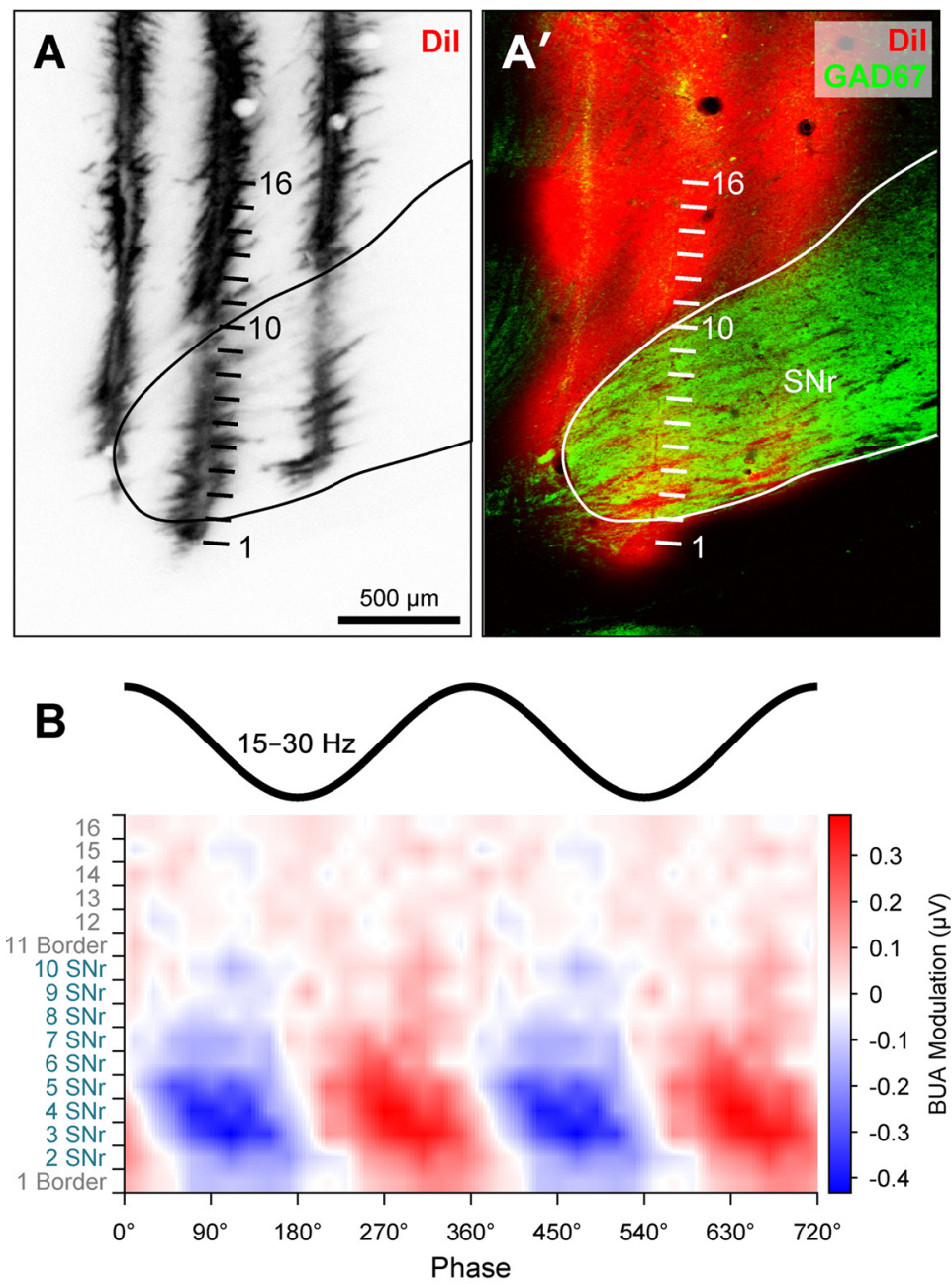

Relative SNr BUA Power (\%)
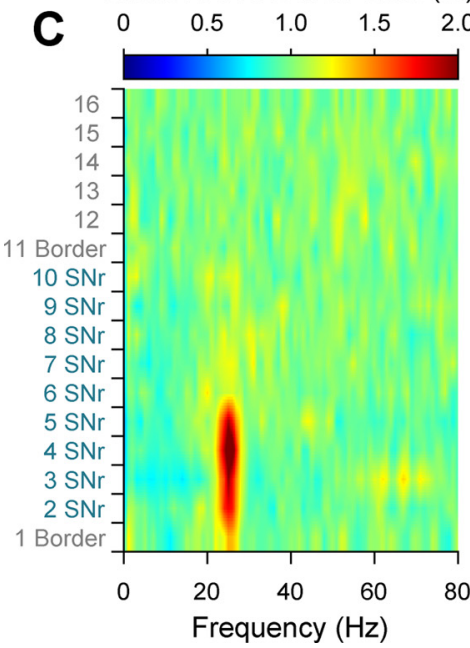

SNr BUA-ECoG Coherence
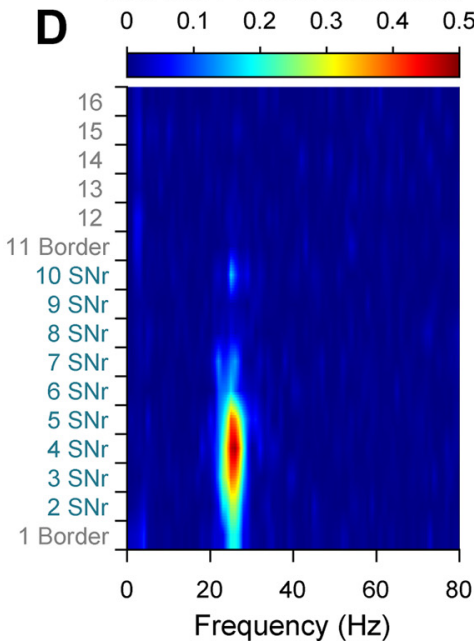

Figure 8. Example localization of BUA signals in the $\mathrm{SNr}$, and their relationship with cortical $\beta$ oscillations. $A$, Image of a parasagittal tissue section from a 6-OHDA-lesioned rat, with Dil fluorescence signal (in an inverted tone for clarity) marking three penetration tracks made at different times by a Dil-coated silicon probe during recordings in vivo. Short lines indicate the estimated positions of the probe's 16 recording contacts along one such track. $\boldsymbol{A}^{\prime}$, The Dil signal (red) was localized with respect to the $\mathrm{SNr}$, as delineated by GAD67 immunofluorescence (green), on the same section as in $\boldsymbol{A}$. Probe contacts 2-10 were considered to have been within SNr. B, BUA signals from the 16 recording contacts at the positions indicated in $\boldsymbol{A} / \boldsymbol{A}^{\prime}$, averaged according to the instantaneous phases of cortical $\beta$ oscillations (bin size $=20^{\circ}$ ) and plotted as a heat map. Note the large-amplitude phasic modulation of BUA signals at contacts 2-6 within the SNr. C, Power spectra of BUA signals, from the same recordings as in $\boldsymbol{B}$, shown as a heat map. Power is relative to that at $1-100 \mathrm{~Hz}$. $\boldsymbol{D}$, Spectra of coherence between the same BUA signals and the simultaneously recorded ECOG. Note the focal nature of SNr power and coherence at $\beta$ frequencies. Bin size of spectra in $C$ and $\boldsymbol{D}$ is $1 \mathrm{~Hz}$. In the parasagittal section shown in $A / A^{\prime}$, rostral is toward the left, and dorsal is toward the top. 

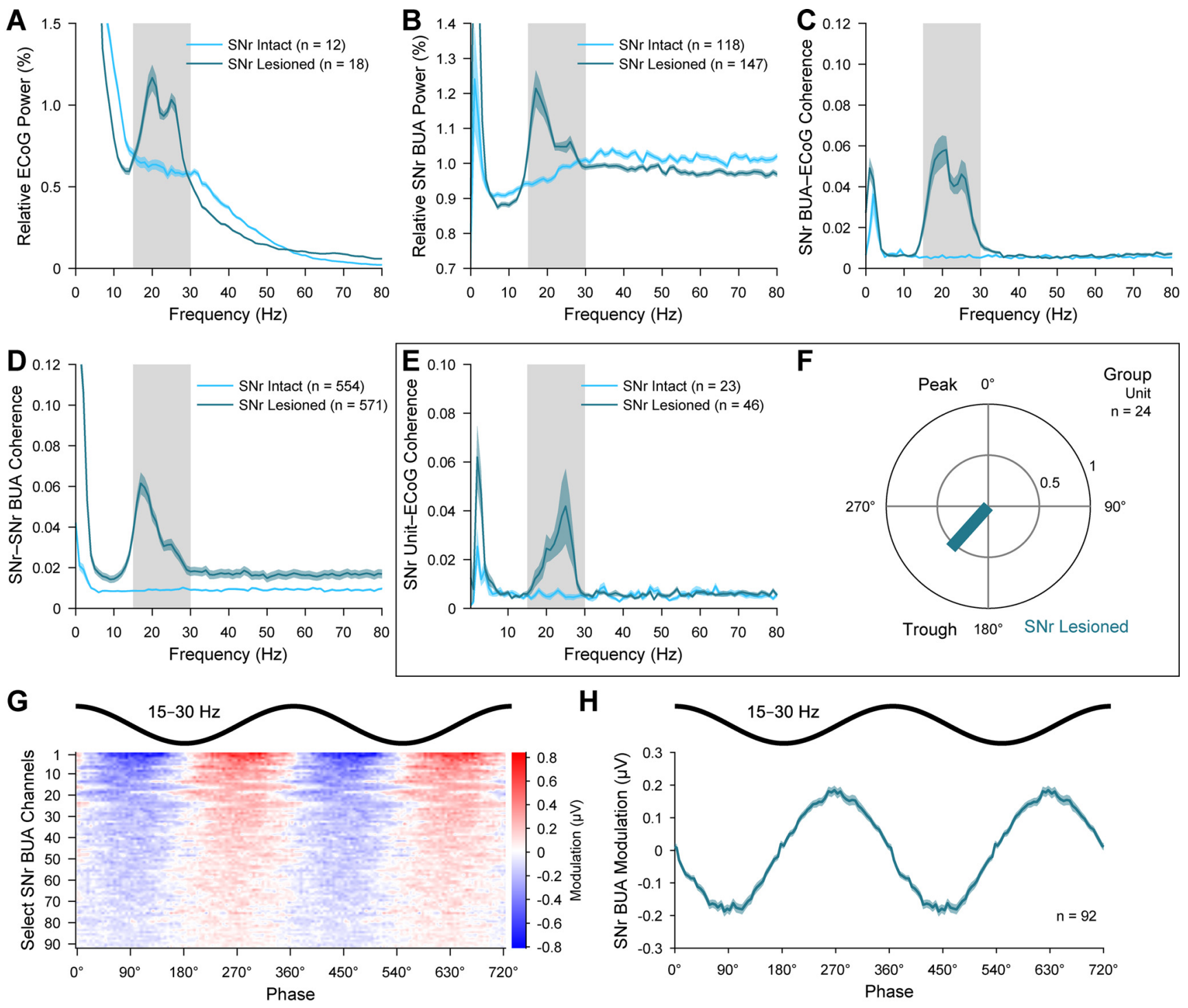

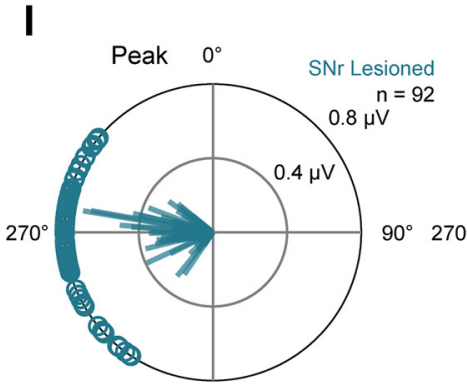

Trough $180^{\circ}$

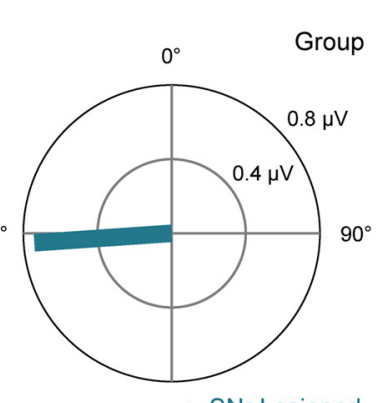

$180^{\circ} \mathrm{SNr}$ Lesioned
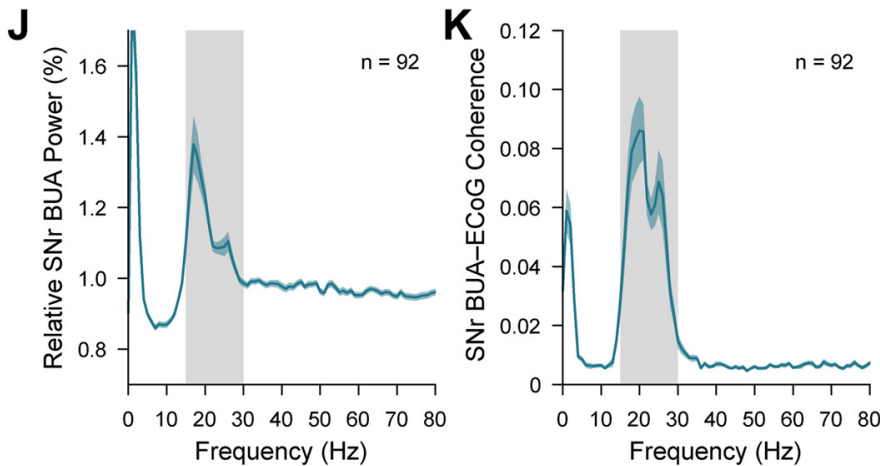

Figure 9. BUA signals in the SNr during cortical activation in dopamine-intact and 6-OHDA-lesioned rats. $A$, Mean power spectra of ECoGs simultaneously recorded with BUA in the SNr in dopamine-intact and 6-OHDA-lesioned rats. Gray shading represents frequency band of $\beta$ oscillations analyzed (15-30 Hz). $\boldsymbol{B}$, Mean power spectra of BUA signals recorded in SNr. Note the peak in power at $\beta$ frequencies for the SNr BUA signals in lesioned rats. $\boldsymbol{C}$, Mean coherence spectra between the SNr BUA signals and ECoGs (color coding and sample sizes as in $\boldsymbol{B}$ ). Note the peak in coherence at $\beta$ frequencies for SNr BUA signals in lesioned rats. $\boldsymbol{D}$, Coherence spectra of simultaneously recorded pairs of SNr BUA signals. $\boldsymbol{E}$, Mean coherence spectra between SNr single units and ECOGs. $\boldsymbol{F}$, Mean vector of the preferred phases of SNr single units in lesioned rats. $\mathbf{G}$, Heat map representation of the phase-averaged waveforms of the significantly modulated SNr BUA signals ( $n=92$ ) in lesioned rats (bin size $=5^{\circ}$; BUA signals sorted by vector length). $\boldsymbol{H}$, Group average of waveforms shown in $\boldsymbol{G}$. Positive modulations of SNr BUA tended to occur during the ascending phase of cortical $\beta$ oscillations. $I$, Left, Circular plots of the individual significantly modulated SNr BUA signals in lesioned rats. Right, Mean vector of the preferred phases of all significantly modulated SNr BUA signals. J, Mean power spectrum of the significantly modulated SNr BUA signals. $\boldsymbol{K}$, Mean coherence spectrum between significantly modulated SNr BUA signals and the simultaneously recorded ECoGs. $\boldsymbol{A}-\boldsymbol{E}, \boldsymbol{H}, \boldsymbol{J}, \boldsymbol{K}$, Data are mean \pm SEM. Bin size of power and coherence spectra is $1 \mathrm{~Hz}$. $n$, the number of individual ECoG recordings $(\boldsymbol{A})$, the number of individual BUA signals recorded $(\boldsymbol{B}, \boldsymbol{C})$, the number of pairs of BUA signals recorded $(\boldsymbol{D})$, the number of single units $(\boldsymbol{E})$, the number of significantly phase-locked single units $(\boldsymbol{F})$, and the number of the significantly modulated SNr BUA signals $(\boldsymbol{H}-\boldsymbol{K})$. All BUA signals and single units were recorded with silicon probes. 

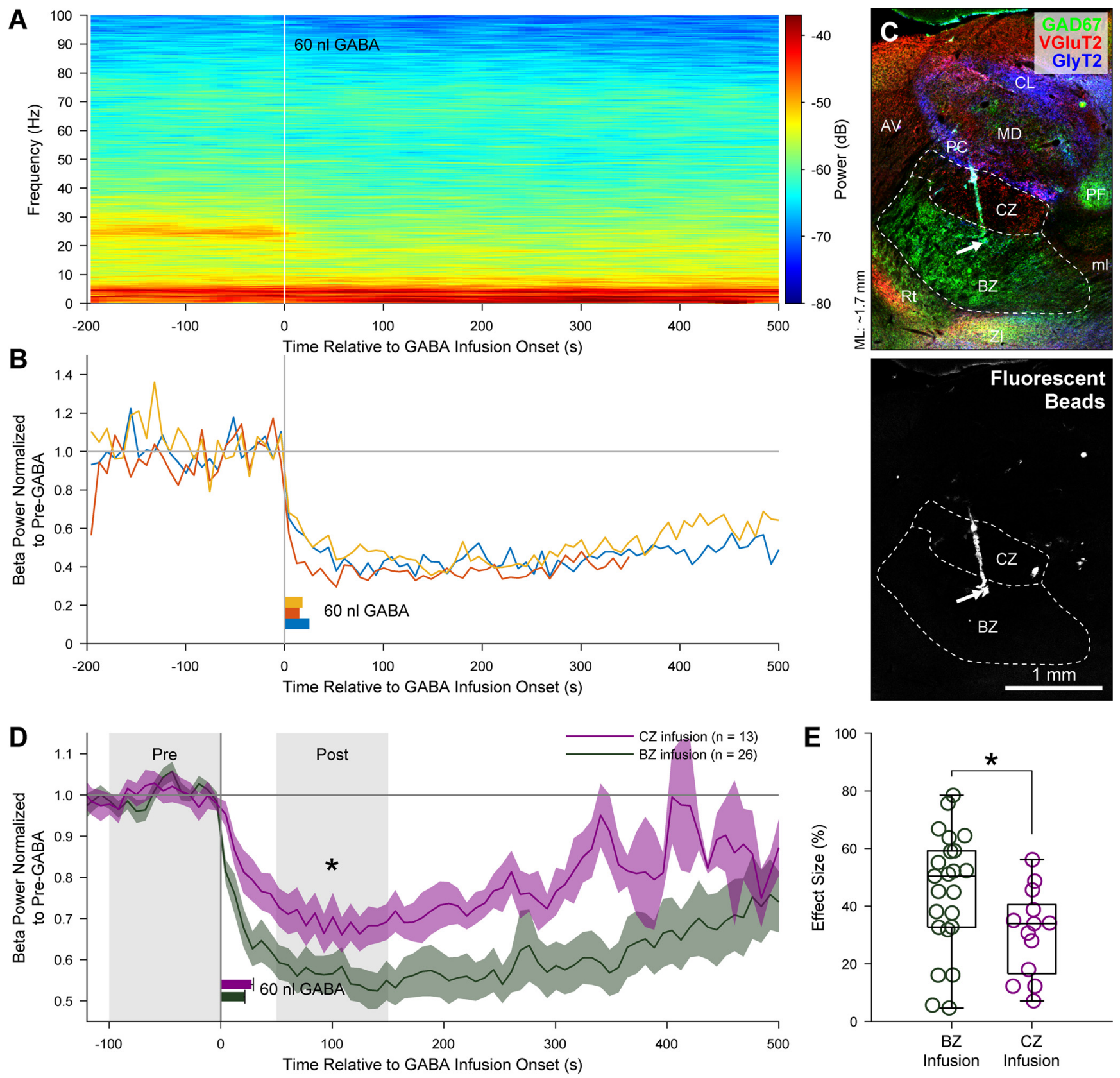

$\mathbf{F}$
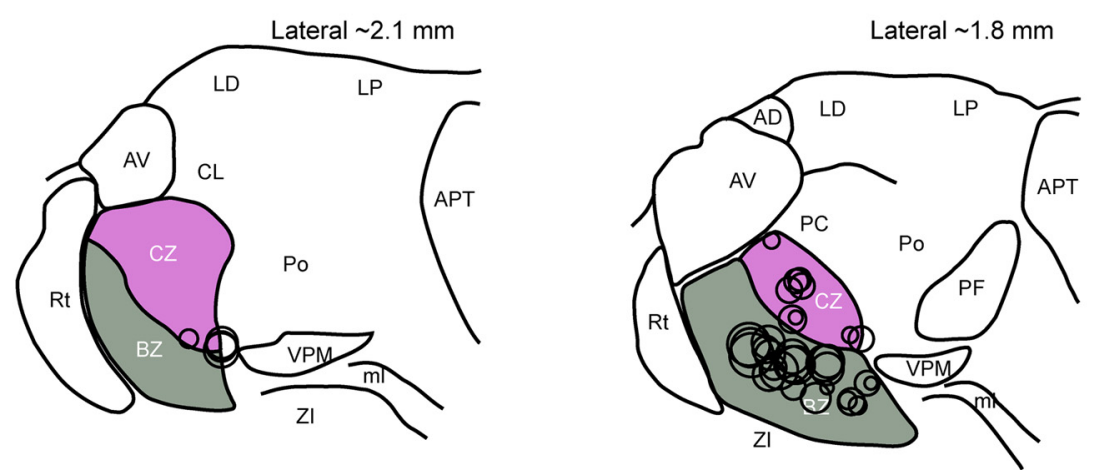

○ $75 \%$

$50 \%$

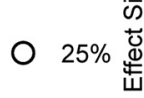

$\circ \quad 0 \%$

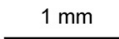

Figure 10. Effects of microinfusions of GABA into motor thalamus on the expression of cortical $\beta$ oscillations in 6-OHDA-lesioned rats. $\boldsymbol{A}$, Time-evolving power spectrogram of oscillations recorded over the motor cortex in a lesioned rat before, during, and after a single infusion of a GABA solution $(60 \mathrm{nl}, 0.5 \mathrm{~m})$ into the BZ of the motor thalamus. Note the clear reduction in the power of the abnormal $\beta$ oscillations (center frequency of $\sim 24 \mathrm{~Hz}$ ) a few seconds after the onset of the GABA infusion (at time $=0 \mathrm{~s}$ ). $\boldsymbol{B}$, Power of cortical oscillations in the $\beta$-frequency band (15-30 Hz; normalized to beta power in the $100 \mathrm{~s}$ immediately preceding GABA infusion) before, during, and after three separate infusions of GABA at the same site in BZ (yellow, red, and blue traces). Blue trace corresponds to the data shown in $A$. The interval between each infusion was $>10 \mathrm{~min}$. Horizontal bars (yellow, red, blue) represent the corresponding duration of 
Abdi et al., 2015; Sharott et al., 2017). We determined that, regardless of two extreme brain states, the spontaneous firing rates of $\mathrm{BZ}$ neurons were not abnormally decreased. Our results agree with electrophysiological studies of $\mathrm{BZ}$ neurons in awake dopamine-intact and Parkinsonian monkeys (Pessiglione et al., 2005; Kammermeier et al., 2016), suggesting that they generalize beyond anesthesia and across species. Ex vivo BZ neurons exhibit augmented "rebound" LTS bursting after dopamine depletion (Bichler et al., 2021). Our data show that this does not manifest as altered propensities to fire spikes in LTS bursts in vivo. That BZ neurons were not hypoactive tallies with our observation that $\mathrm{SNr}$ neurons were not hyperactive after dopamine depletion; the latter is a recurring finding in awake and anesthetized rodents (Tseng et al., 2005; Walters et al., 2007; Lobb and Jaeger, 2015; Willard et al., 2019). We observed, however, that $\mathrm{CZ}$ neurons had increased firing rates during cortical activation, adding to evidence of altered CZ activity in Parkinsonism (Galvan et al., 2015; Wichmann, 2019). This CZ hyperactivity might reflect augmented output from (presumably glutamatergic) neurons in cerebellar nuclei after dopamine depletion (Menardy et al., 2019). Our recordings in BZ do not uphold canonical firing rate-based models of BG-thalamocortical dysfunction in Parkinsonism, but the predicted alterations might still emerge during specific motor behaviors. Nevertheless, our data support and extend the concept that profoundly dysrhythmic activity can arise without firing rate changes in the Parkinsonian BZ and SNr.

\section{Dysrhythmia in the Parkinsonian motor thalamus during cortical SWA}

Previous studies of BG neurons show that dopamine depletion can alter the patterning of their discharges during, and with respect to, the stereotyped cortical slow oscillation, thereby providing valuable insights into the potential contributions of different sets of inputs to their activity (Magill et al., 2001; Belluscio et al., 2003; Tseng et al., 2005; Mallet et al., 2006; Walters et al., 2007; Zold et al., 2007, 2012; Abdi et al., 2015; Sharott et al., 2017). An extreme example of altered activity patterning occurs in the external globus pallidus; the firing of prototypic neurons in dopamine-intact animals tends to increase slightly around the

\section{$\leftarrow$}

each manually controlled GABA infusion. Note the highly reproducible time course and magnitude of the reduction in cortical beta power after GABA infusion. $C$, The GABA infusion site accessed in $\boldsymbol{A}$ and $\boldsymbol{B}$ was localized to the $B Z$ of motor thalamus by post hoc anatomicical analyses; the fluorescent beads marking the trajectory of the infusion pipette traversed the border between $C Z$ and $B Z$ (dashed line), and then terminated in the BZ (arrows). $D$, Mean power of cortical $\beta$ oscillations before, during, and after all infusions of GABA at BZ sites (green trace) or at $\mathrm{CZ}$ sites (purple trace) in the motor thalamus. Green and purple shaded areas represent SEMs. Horizontal bars represent the mean durations (+ SEM) of the manually controlled GABA infusions into $B Z$ (green) or $C Z$ (purple). The nadirs in cortical beta power occurred at $\sim 100 \mathrm{~s}$ after the onset of GABA infusion. The cortical beta power at 50 $150 \mathrm{~s}$ after $B Z$ infusions was lower than that after $C Z$ infusions. $E$, Effect sizes of all GABA infusions at BZ sites (green circles) or CZ sites (purple circles). Effect size was defined as the percentage reduction in cortical beta power at 50-150 s after GABA infusion ("Post" gray shading in D) compared with power during the $100 \mathrm{~s}$ immediately before infusion ("Pre" gray shading in $\boldsymbol{D}$ ). On average, the effect sizes of $\mathrm{BZ}$ infusions were larger than those of $\mathrm{CZ}$ infusions ( ${ }^{*} p=0.038$, MWUT). $\boldsymbol{F}$, Effect size of each GABA infusion according to its location in $B Z$ and $C Z$. In the parasagittal sections shown in $\boldsymbol{C}$ and $\boldsymbol{F}$, rostral is toward the left, and dorsal is toward the top. APT, anterior pretectal nucleus; LD, lateral dorsal nucleus; LP, lateral posterior nucleus, ml, medial lemniscus; PF, parafascicular nucleus. For abbreviations of other structures in and around the thalamus, see Figures 1 and 6. peaks of cortical slow oscillations, whereas prototypic neuron firing in 6-OHDA-lesioned animals is strongly timed with slow oscillation troughs (Mallet et al., 2008a, 2012; Abdi et al., 2015). This aberrant "antiphase" oscillatory firing of prototypic neurons in Parkinsonism is likely the result of receiving hypersynchronous rhythmic GABAergic inputs from striatal neurons (Zold et al., 2012; Nevado-Holgado et al., 2014; Sharott et al., 2017; Kovaleski et al., 2020). It follows that hypersynchronous rhythmic GABAergic outputs from BG should aberrantly entrain thalamic neurons, such that their preferred phase of firing is disturbed (Tseng, 2009). One previous study has addressed this prediction, revealing that neurons in the thalamic parafascicular nucleus, also targeted by GABAergic BG outputs, do not exhibit the expected antiphase firing (Parr-Brownlie et al., 2009). In stark contrast, we demonstrate here that this prediction holds true for $\mathrm{BZ}$ neurons, with dopamine depletion resulting in weaker phase-locking and a $\sim 100^{\circ}$ shift in the mean angles of their firing with respect to cortical slow oscillations. Notably, the phase-locked firing of $\mathrm{CZ}$ neurons was not inappropriately timed to slow oscillations, reinforcing that SWA-related dysrhythmia in the Parkinsonian motor thalamus is selective for input zone. In line with other studies (Belluscio et al., 2003; Tseng et al., 2005; Walters et al., 2007; Lobb and Jaeger, 2015), the temporal coupling of $\mathrm{SNr}$ neuron firing to cortical slow oscillations was markedly stronger in lesioned rats, such that some $\mathrm{SNr}$ neurons fired in a phasic bursting manner. We determined that $\mathrm{SNr}$ burst onsets timed with cyclical reductions in BZ neuron firing, whereas $\mathrm{SNr}$ burst offsets timed with the primary peak of $\mathrm{BZ}$ neuron activity. As such, the aberrant phase-locked burst firing of $\mathrm{SNr}$ neurons appears well suited to mediate the phase shift and dysrhythmia of BZ neurons after dopamine depletion; in future studies, it would be important to progress from correlation to causation. Ascribing this role to $\mathrm{SNr}$ does not preclude the possibility that other inputs to $\mathrm{BZ}$, such as those from cortex, play important roles in driving the dysrhythmic activity of $\mathrm{BZ}$ neurons. In turn, the dysrhythmic firing of $\mathrm{BZ}$ neurons would be broadcast to wide areas of frontal cortex (Herkenham, 1979; Kuramoto et al., 2009, 2015), wherein it might negatively impact on activity dynamics. Numerous studies in people with PD have demonstrated alterations in cortical SWA and slow-wave sleep, with further implications for symptoms and quality of life (Zahed et al., 2021).

\section{Dysrhythmia in the Parkinsonian motor thalamus during cortical activation}

We observed that individual BZ neurons inappropriately engage with the exaggerated cortical $\beta$ oscillations that arise during activated brain states after dopamine depletion. This agrees with a study of the VM nucleus in awake 6-OHDA-lesioned rats (Brazhnik et al., 2016). It should be noted, however, that VM is difficult to objectively delineate with Nissl staining (as used in Brazhnik et al., 2016), and that VM is only a fraction of the BZ (Nakamura et al., 2014). Here, we provide important advances by accurately localizing recordings to the $\mathrm{BZ}$ and $\mathrm{CZ}$ (thus addressing selectivity for input zone), by defining the extent to which BZ neuronal ensembles are rhythmically synchronized, and by determining whether engagement in $\beta$ oscillations is accompanied by changes in $\mathrm{BZ}$ neuron firing rates. Our analyses of BUA signals show that, after dopamine depletion, focally organized neuronal ensembles in the BZ inappropriately synchronize their outputs at $\beta$ frequencies. It was not possible to determine whether there was a phase shift in BZ neuron firing during cortical activation, as occurred during SWA, because so 
few neurons coupled their firing to the weak/transient cortical $\beta$ oscillations present in the dopamine-intact state. These experiments reveal a second manifestation of dysrhythmia in the Parkinsonian motor thalamus, and we reiterate that exaggerated $\beta$ oscillations arise in $\mathrm{BZ}$ without firing rate changes. Expression of pathological $\beta$-frequency activities is exquisitely selective for input zone, such that CZ neurons are not similarly dysrhythmic. Studies of idiopathic PD support the notion that exaggerated beta-band synchronization of BG neuronal activity underpins bradykinesia/rigidity (Kühn et al., 2006, 2009; Ray et al., 2008; Sharott et al., 2014, 2018). We conclude that BZ neurons are primed to mediate the detrimental influences of abnormal betaband activity on neuronal information processing and movement in PD.

We observed that the temporal coupling of cortical $\beta$ oscillations to the firing of individual SNr neurons was markedly stronger after dopamine depletion, as in awake rats (Brazhnik et al., 2012, 2014). Extending our analysis to BUA signals, we detail the novel observation that focal neuronal ensembles in the $\mathrm{SNr}$ inappropriately synchronize their outputs at $\beta$ frequencies. Importantly, there was an approximate antiphase relationship between $\mathrm{SNr}$ and BZ ensemble activities at $\beta$ frequencies; on average, peaks in $\mathrm{SNr}$ ensemble activity preceded peaks in $\mathrm{BZ}$ ensemble activity by $\sim 217^{\circ}$. Given a range of beta oscillation periods of $\sim 33-66 \mathrm{~ms}$, this phase difference would represent a time delay of $\sim 20-40 \mathrm{~ms}$, which tallies with the time course of evoked nigrothalamic IPSPs and the subsequent pauses they cause in BZ neuron firing ex vivo (Edgerton and Jaeger, 2014). Together, our data suggest that aberrant inhibitory outputs arising from the hypersynchronized beta-band firing of SNr neurons are a valid candidate for orchestrating the related dysrhythmia of BZ neurons in Parkinsonism. Again, in future studies, it would be important to address causation.

Frontal cortical areas innervating motor thalamus exhibit exaggerated $\beta$ oscillations after dopamine depletion, as evidenced in our ECoG recordings here as well as those in awake rats (Sharott et al., 2005; Mallet et al., 2008b; Li et al., 2012; Brazhnik et al., 2016). It is thus possible that cortex also directly entrains $\mathrm{BZ}$ neurons to $\beta$ rhythms. However, it is unknown whether cortical neurons innervating BZ (and/or CZ) engage in abnormal $\beta$ oscillations. In terms of reciprocal influence, our GABA microinfusion experiments strongly suggest that ongoing activity in the $\mathrm{BZ}$ in particular bolsters exaggerated $\beta$ oscillations in motor cortex. This would fit well with BZ neurons being positioned, via extensive axon collateral networks (Kuramoto et al., 2009, 2015), to deliver beta-band inputs to many cortical neurons. We conclude that the dysrhythmic BZ is a critical node in the wider BGthalamocortical loop circuit for the generation and/or maintenance of pathological cortical $\beta$ oscillations in PD.

\section{References}

Abdi A, Mallet N, Mohamed FY, Sharott A, Dodson PD, Nakamura KC, Suri S, Avery SV, Larvin JT, Garas FN, Garas SN, Vinciati F, Morin S, Bezard E, Baufreton J, Magill PJ (2015) Prototypic and arkypallidal neurons in the dopamine-intact external globus pallidus. J Neurosci 35:6667-6688.

Anderson CJ, Sheppard DT, Huynh R, Anderson DN, Polar CA, Dorval AD (2015) Subthalamic deep brain stimulation reduces pathological information transmission to the thalamus in a rat model of parkinsonism. Front Neural Circuits 9:31.

Anderson ME, Turner RS (1991) Activity of neurons in cerebellar-receiving and pallidal-receiving areas of the thalamus of the behaving monkey. $J$ Neurophysiol 66:879-893.
Avila I, Parr-Brownlie LC, Brazhnik E, Castañeda E, Bergstrom DA, Walters JR (2010) Beta frequency synchronization in basal ganglia output during rest and walk in a hemiparkinsonian rat. Exp Neurol 221:307-319.

Basha D, Dostrovsky JO, Lopez Rios AL, Hodaie M, Lozano AM, Hutchison WD (2014) Beta oscillatory neurons in the motor thalamus of movement disorder and pain patients. Exp Neurol 261:782-790.

Belluscio MA, Kasanetz F, Riquelme LA, Murer MG (2003) Spreading of slow cortical rhythms to the basal ganglia output nuclei in rats with nigrostriatal lesions. Eur J Neurosci 17:1046-1052.

Berens P (2009) CircStat: a MATLAB toolbox for circular statistics. J Stat Softw 31:1-21.

Bichler EK, Cavarretta F, Jaeger D (2021) Changes in excitability properties of ventromedial motor thalamic neurons in 6-OHDA lesioned mice. eNeuro 8:ENEURO.0436-20.2021.

Bosch-Bouju C, Hyland BI, Parr-Brownlie LC (2013) Motor thalamus integration of cortical, cerebellar and basal ganglia information: implications for normal and parkinsonian conditions. Front Comput Neurosci 7:163.

Bosch-Bouju C, Smither RA, Hyland BI, Parr-Brownlie LC (2014) Reduced reach-related modulation of motor thalamus neural activity in a rat model of Parkinson's disease. J Neurosci 34:15836-15850.

Brazhnik E, Cruz AV, Avila I, Wahba MI, Novikov N, Ilieva NM, McCoy AJ, Gerber C, Walters JR (2012) State-dependent spike and local field synchronization between motor cortex and substantia nigra in hemiparkinsonian rats. J Neurosci 32:7869-7880.

Brazhnik E, Novikov N, McCoy AJ, Cruz AV, Walters JR (2014) Functional correlates of exaggerated oscillatory activity in basal ganglia output in hemiparkinsonian rats. Exp Neurol 261:563-577.

Brazhnik E, McCoy AJ, Novikov N, Hatch CE, Walters JR (2016) Ventral medial thalamic nucleus promotes synchronization of increased high beta oscillatory activity in the basal ganglia-thalamocortical network of the hemiparkinsonian rat. J Neurosci 36:4196-4208.

Chauvette S, Volgushev M, Timofeev I (2010) Origin of active states in local neocortical networks during slow sleep oscillation. Cereb Cortex 20:2660-2674.

DeLong MR (1990) Primate models of movement disorders of basal ganglia origin. Trends Neurosci 13:281-285.

Devergnas A, Chen E, Ma Y, Hamada I, Pittard D, Kammermeier S, Mullin AP, Faundez V, Lindsley CW, Jones C, Smith Y, Wichmann T (2016) Anatomical localization of Cav3.1 calcium channels and electrophysiological effects of T-type calcium channel blockade in the motor thalamus of MPTP-treated monkeys. J Neurophysiol 115:470-485.

Edgerton JR, Jaeger D (2014) Optogenetic activation of nigral inhibitory inputs to motor thalamus in the mouse reveals classic inhibition with little potential for rebound activation. Front Cell Neurosci 8:36.

Fanselow EE, Sameshima K, Baccala LA, Nicolelis MA (2001) Thalamic bursting in rats during different awake behavioral states. Proc Natl Acad Sci USA 98:15330-15335.

Fisher NI (1995) Statistical analysis of circular data. Cambridge: Cambridge UP.

Galvan A, Devergnas A, Wichmann T (2015) Alterations in neuronal activity in basal ganglia-thalamocortical circuits in the parkinsonian state. Front Neuroanat 9:5.

Garas FN, Shah RS, Kormann E, Doig NM, Vinciati F, Nakamura KC, Dorst MC, Smith Y, Magill PJ, Sharott A (2016) Secretagogin expression delineates functionally-specialized populations of striatal parvalbumin-containing interneurons. Elife 5:e16088.

Guehl D, Pessiglione M, François C, Yelnik J, Hirsch EC, Féger J, Tremblay L (2003) Tremor-related activity of neurons in the 'motor' thalamus: changes in firing rate and pattern in the MPTP vervet model of parkinsonism. Eur J Neurosci 17:2388-2400.

Hammond C, Bergman H, Brown P (2007) Pathological synchronization in Parkinson's disease: networks, models and treatments. Trends Neurosci 30:357-364

Herkenham M (1979) The afferent and efferent connections of the ventromedial thalamic nucleus in the rat. J Comp Neurol 183:487-517.

Hioki H, Fujiyama F, Taki K, Tomioka R, Furuta T, Tamamaki N, Kaneko T (2003) Differential distribution of vesicular glutamate transporters in the rat cerebellar cortex. Neuroscience 117:1-6.

Holt GR, Softky WR, Koch C, Douglas RJ (1996) Comparison of discharge variability in vitro and in vivo in cat visual cortex neurons. J Neurophysiol 75:1806-1814. 
Journée HL (1983) Demodulation of amplitude modulated noise: a mathematical evaluation of a demodulator for pathological tremor EMG's. IEEE Trans Biomed Eng 30:304-308.

Kammermeier S, Pittard D, Hamada I, Wichmann T (2016) Effects of highfrequency stimulation of the internal pallidal segment on neuronal activity in the thalamus in parkinsonian monkeys. J Neurophysiol 116:28692881.

Kojima S, Doupe AJ (2009) Activity propagation in an avian basal gangliathalamocortical circuit essential for vocal learning. J Neurosci 29:47824793.

Kovaleski RF, Callahan JW, Chazalon M, Wokosin DL, Baufreton J, Bevan MD (2020) Dysregulation of external globus pallidus-subthalamic nucleus network dynamics in parkinsonian mice during cortical slow-wave activity and activation. J Physiol 598:1897-1927.

Kühn AA, Doyle L, Pogosyan A, Yarrow K, Kupsch A, Schneider GH, Hariz MI, Trottenberg T, Brown P (2006) Modulation of beta oscillations in the subthalamic area during motor imagery in Parkinson's disease. Brain 129:695-706.

Kühn AA, Tsui A, Aziz T, Ray N, Brücke C, Kupsch A, Schneider GH, Brown P (2009) Pathological synchronisation in the subthalamic nucleus of patients with Parkinson's disease relates to both bradykinesia and rigidity. Exp Neurol 215:380-387.

Kuramoto E, Furuta T, Nakamura KC, Unzai T, Hioki H, Kaneko T (2009) Two types of thalamocortical projections from the motor thalamic nuclei of the rat: a single neuron-tracing study using viral vectors. Cereb Cortex 19:2065-2077.

Kuramoto E, Fujiyama F, Nakamura KC, Tanaka Y, Hioki H, Kaneko T (2011) Complementary distribution of glutamatergic cerebellar and GABAergic basal ganglia afferents to the rat motor thalamic nuclei. Eur J Neurosci 33:95-109.

Kuramoto E, Ohno S, Furuta T, Unzai T, Tanaka YR, Hioki H, Kaneko T (2015) Ventral medial nucleus neurons send thalamocortical afferents more widely and more preferentially to layer 1 than neurons of the ventral anterior-ventral lateral nuclear complex in the rat. Cereb Cortex 25:221-235.

Lacey CJ, Bolam JP, Magill PJ (2007) Novel and distinct operational principles of intralaminar thalamic neurons and their striatal projections. J Neurosci 27:4374-4384.

Lachaux JP, Rodriguez E, Martinerie J, Varela FJ (1999) Measuring phase synchrony in brain signals. Hum Brain Mapp 8:194-208.

Li Q, Ke Y, Chan DC, Qian ZM, Yung KK, Ko H, Arbuthnott GW, Yung WH (2012) Therapeutic deep brain stimulation in Parkinsonian rats directly influences motor cortex. Neuron 76:1030-1041.

Lobb CJ, Jaeger D (2015) Bursting activity of substantia nigra pars reticulata neurons in mouse parkinsonism in awake and anesthetized states. Neurobiol Dis 75:177-185.

Lu SM, Guido W, Sherman SM (1992) Effects of membrane voltage on receptive field properties of lateral geniculate neurons in the cat: contributions of the low-threshold $\mathrm{Ca}^{2+}$ conductance. J Neurophysiol 68:2185-2198.

Magill PJ, Bolam JP, Bevan MD (2001) Dopamine regulates the impact of the cerebral cortex on the subthalamic nucleus-globus pallidus network. Neuroscience 106:313-330.

Magill PJ, Pogosyan A, Sharott A, Csicsvari J, Bolam JP, Brown P (2006) Changes in functional connectivity within the rat striatopallidal axis during global brain activation in vivo. J Neurosci 26:6318-6329.

Mallet N, Ballion B, Le Moine C, Gonon F (2006) Cortical inputs and GABA interneurons imbalance projection neurons in the striatum of parkinsonian rats. J Neurosci 26:3875-3884.

Mallet N, Pogosyan A, Márton LF, Bolam JP, Brown P, Magill PJ (2008a) Parkinsonian beta oscillations in the external globus pallidus and their relationship with subthalamic nucleus activity. J Neurosci 28:1424514258.

Mallet N, Pogosyan A, Sharott A, Csicsvari J, Bolam JP, Brown P, Magill PJ (2008b) Disrupted dopamine transmission and the emergence of exaggerated beta oscillations in subthalamic nucleus and cerebral cortex. J Neurosci 28:4795-4806.

Mallet N, Micklem BR, Henny P, Brown MT, Williams C, Bolam JP, Nakamura KC, Magill PJ (2012) Dichotomous organization of the external globus pallidus. Neuron 74:1075-1086.

Menardy F, Varani AP, Combes A, Léna C, Popa D (2019) Functional alteration of cerebello-cerebral coupling in an experimental mouse model of Parkinson's disease. Cereb Cortex 29:1752-1766.
Moran A, Bar-Gad I (2010) Revealing neuronal functional organization through the relation between multi-scale oscillatory extracellular signals. J Neurosci Methods 186:116-129.

Moran A, Bergman H, Israel Z, Bar-Gad I (2008) Subthalamic nucleus functional organization revealed by parkinsonian neuronal oscillations and synchrony. Brain 131:3395-3409.

Myers LJ, Lowery M, O’Malley M, Vaughan CL, Heneghan C, St Clair Gibson A, Harley YX, Sreenivasan R (2003) Rectification and non-linear pre-processing of EMG signals for cortico-muscular analysis. J Neurosci Methods 124:157-165.

Nakamura K, Morrison SF (2007) Central efferent pathways mediating skin cooling-evoked sympathetic thermogenesis in brown adipose tissue. Am J Physiol Regul Integr Comp Physiol 292:R127-R136.

Nakamura KC, Sharott A, Magill PJ (2014) Temporal coupling with cortex distinguishes spontaneous neuronal activities in identified basal gangliarecipient and cerebellar-recipient zones of the motor thalamus. Cereb Cortex 24:81-97.

Nevado-Holgado AJ, Mallet N, Magill PJ, Bogacz R (2014) Effective connectivity of the subthalamic nucleus-globus pallidus network during Parkinsonian oscillations. J Physiol 592:1429-1455.

Parr-Brownlie LC, Poloskey SL, Bergstrom DA, Walters JR (2009) Parafascicular thalamic nucleus activity in a rat model of Parkinson's disease. Exp Neurol 217:269-281.

Paxinos G, Watson C (2007) The rat brain in stereotaxic coordinates, Ed 6. London: Academic.

Pessiglione M, Guehl D, Rolland AS, François C, Hirsch EC, Féger J, Tremblay L (2005) Thalamic neuronal activity in dopamine-depleted primates: evidence for a loss of functional segregation within basal ganglia circuits. J Neurosci 25:1523-1531.

Ray NJ, Jenkinson N, Wang S, Holland P, Brittain JS, Joint C, Stein JF, Aziz T (2008) Local field potential beta activity in the subthalamic nucleus of patients with Parkinson's disease is associated with improvements in bradykinesia after dopamine and deep brain stimulation. Exp Neurol 213:108-113.

Rubin JE, McIntyre CC, Turner RS, Wichmann T (2012) Basal ganglia activity patterns in parkinsonism and computational modeling of their downstream effects. Eur J Neurosci 36:2213-2228.

Sakata S, Harris KD (2009) Laminar structure of spontaneous and sensoryevoked population activity in auditory cortex. Neuron 64:404-418.

Schneider JS, Rothblat DS (1996) Alterations in intralaminar and motor thalamic physiology following nigrostriatal dopamine depletion. Brain Res 742:25-33.

Schwarting RK, Huston JP (1996) Unilateral 6-hydroxydopamine lesions of meso-striatal dopamine neurons and their physiological sequelae. Prog Neurobiol 49:215-266.

Sharott A, Magill PJ, Harnack D, Kupsch A, Meissner W, Brown P (2005) Dopamine depletion increases the power and coherence of beta-oscillations in the cerebral cortex and subthalamic nucleus of the awake rat. Eur J Neurosci 21:1413-1422.

Sharott A, Doig NM, Mallet N, Magill PJ (2012) Relationships between the firing of identified striatal interneurons and spontaneous and driven cortical activities in vivo. J Neurosci 32:13221-13236.

Sharott A, Gulberti A, Zittel S, Tudor Jones AA, Fickel U, Münchau A, Köppen JA, Gerloff C, Westphal M, Buhmann C, Hamel W, Engel AK, Moll CK (2014) Activity parameters of subthalamic nucleus neurons selectively predict motor symptom severity in Parkinson's disease. J Neurosci 34:6273-6285.

Sharott A, Vinciati F, Nakamura KC, Magill PJ (2017) A population of indirect pathway striatal projection neurons is selectively entrained to Parkinsonian beta oscillations. J Neurosci 37:9977-9998.

Sharott A, Gulberti A, Hamel W, Köppen JA, Münchau A, Buhmann C, Pötter-Nerger M, Westphal M, Gerloff C, Moll CK, Engel AK (2018) Spatio-temporal dynamics of cortical drive to human subthalamic nucleus neurons in Parkinson's disease. Neurobiol Dis 112:49-62.

Siapas AG, Lubenov EV, Wilson MA (2005) Prefrontal phase locking to hippocampal theta oscillations. Neuron 46:141-151.

Smith Y, Bevan MD, Shink E, Bolam JP (1998) Microcircuitry of the direct and indirect pathways of the basal ganglia. Neuroscience 86:353-387.

Steriade M (2000) Corticothalamic resonance, states of vigilance and mentation. Neuroscience 101:243-276.

Szucs A (1998) Applications of the spike density function in analysis of neuronal firing patterns. J Neurosci Methods 81:159-167. 
Tseng KY (2009) Facing the lack of antiphase oscillation in the parafascicular nucleus after dopamine depletion. Exp Neurol 219:62-65.

Tseng KY, Kargieman L, Gacio S, Riquelme LA, Murer MG (2005) Consequences of partial and severe dopaminergic lesion on basal ganglia oscillatory activity and akinesia. Eur J Neurosci 22:2579-2586.

Ushimaru M, Ueta Y, Kawaguchi Y (2012) Differentiated participation of thalamocortical subnetworks in slow/spindle waves and desynchronization. J Neurosci 32:1730-1746.

Vitek JL, Ashe J, DeLong MR, Alexander GE (1994) Physiologic properties and somatotopic organization of the primate motor thalamus. J Neurophysiol 71:1498-1513.

von Nicolai C, Engler G, Sharott A, Engel AK, Moll CK, Siegel M (2014) Corticostriatal coordination through coherent phase-amplitude coupling. J Neurosci 34:5938-5948.

Walters JR, Hu D, Itoga CA, Parr-Brownlie LC, Bergstrom DA (2007) Phase relationships support a role for coordinated activity in the indirect pathway in organizing slow oscillations in basal ganglia output after loss of dopamine. Neuroscience 144:762-776.
Wichmann $T$ (2019) Changing views of the pathophysiology of Parkinsonism. Mov Disord 34:1130-1143.

Willard AM, Isett BR, Whalen TC, Mastro KJ, Ki CS, Mao X, Gittis AH (2019) State transitions in the substantia nigra reticulata predict the onset of motor deficits in models of progressive dopamine depletion in mice. Elife 8:e42746.

Wu T, Hallett M (2013) The cerebellum in Parkinson's disease. Brain 136:696-709.

Zahed H, Zuzuarregui JR, Gilron R, Denison T, Starr PA, Little S (2021) The neurophysiology of sleep in Parkinson's disease. Mov Disord 36:15261542.

Zold CL, Ballion B, Riquelme LA, Gonon F, Murer MG (2007) Nigrostriatal lesion induces D2-modulated phase-locked activity in the basal ganglia of rats. Eur J Neurosci 25:2131-2144.

Zold CL, Escande MV, Pomata PE, Riquelme LA, Murer MG (2012) Striatal NMDA receptors gate cortico-pallidal synchronization in a rat model of Parkinson's disease. Neurobiol Dis 47:38-48. 